\title{
Auctioning a Discrete Public Good under Incomplete Information
}

\author{
Murat Yilmaz ${ }^{* \dagger}$ \\ Boğaziçi University
}

May 2010

\begin{abstract}
This paper considers a natural dynamic auction mechanism in the context of private provision of a discrete public good under incomplete information. There are two bidders with private valuations, and the cost of the public good is common knowledge. No bidder is willing to provide the good on his own. We show that a natural application of open ascending auctions in such environments fails dramatically: The probability of provision is zero in any equilibrium. The mechanism effectively auctions off the "right" to be the last one to contribute, but intuition suggests that neither player wishes to be the last one to contribute. Since the player who contributes first has the advantage of being able to free ride on the contribution of the other player, no player wants to win the auction. In the light of this intuition, we consider an alternative mechanism in which the "right" to contribute first is sold in the first stage, and in the second stage players are playing a sequential contribution game with the order determined in the first stage. We show that under weak conditions, this mechanism weakly outperforms the sequential contribution mechanism with exogenous order, in terms of the probability of provision.
\end{abstract}

Keywords : discrete public good, open-ascending auction, sequential contribution

JEL Classification Numbers: H41, D44, D61

${ }^{*}$ Boğaziçi University, Department of Economics, Bebek, Istanbul, 34342. Email: muraty@boun.edu.tr. Phone: +90 212359 7656. Fax: +90212 287 2453. Web site: http://www.econ.boun.edu.tr/yilmaz/

${ }^{\dagger}$ I am particularly indebted to Bart Lipman for his guidance and support. I am also grateful to Andrew Newman and Sambuddha Ghosh for their advice. I would like to thank Dilip Mookherjee, Jacob Glazer, Linxia Ren and the seminar participants at Boston University for their useful comments. 


\section{Introduction}

Auction mechanisms have proven to be very useful for allocating private goods under asymmetric information. They are also very simple and familiar. Thus it seems natural to consider auction mechanisms as a way of modeling private provision of public goods. In this paper, we study several plausible auction mechanisms for public goods and analyze their efficiency properties.

Of course, the mechanisms considered here cannot improve on the optimal mechanism for the provision of a public good. However, as Ledyard and Palfrey (1999) observe, optimal mechanisms in this setting involve complicated transfer schemes. Hence these mechanisms seem implausible as a description of private provision of public goods. One can respond to this problem by identifying simple and plausibly private mechanisms which achieve the outcome of the optimal mechanism on at least some restricted domain, an approach followed by Barbieri and Malueg (2008). ${ }^{1}$ Alternatively, one can consider simple and plausibly private mechanisms which perform well globally. We adopt the latter approach and consider the use of auctions as a mechanism for private provision of public goods under incomplete information.

We focus on discrete public goods where the provision decision is only whether or not to produce the good. A street light, public radio fund-raising to finance a certain program, a toll-free bridge are typical examples of a discrete public good. The cost of providing the good is publicly known and each agent has a privately known valuation for the good. We show that there is no provision in a natural adaptation of open ascending auctions where drop out prices are the contributions. In the light of this result, we propose an alternative mechanism in which the "right" to contribute first is auctioned off before the contributors play a sequential contribution game and show that under weak conditions, this mechanism performs better than the well-known sequential contribution mechanism with exogenous order. $^{2}$

In the open ascending auction mechanism we consider, each bidder, observing the ascending price and whether the other bidder has dropped out or not, drops out at his preferred price. A bidder's contribution is his drop out price; that is, we have an all-pay feature. Otherwise, there is no hope for

\footnotetext{
${ }^{1}$ They show that, restricting attention to piecewise-linear equilibrium, the subscription game, a contribution game with full refunds when there is no provision, achieves the outcome of the optimal mechanism.

${ }^{2}$ The contributions towards a discrete public good or a project of common interest are often collected according to a given order. The donors are contacted following an order to collect their contributions to charities during fundraising. In 1995, the Governor of Wisconsin offered $\$ 27$ million to finance a new $\$ 72$ million basketball arena for the University of Wisconsin, on the condition that the rest of the money be raised by private donations. That is, the Governor was the first mover and the private donations were the second.
} 
provision simply because no bidder is willing to provide the good on his own. If the contributions add up to a level at least as big as the cost of the public good, then the good is provided. Otherwise there is no provision and no payments are made. ${ }^{3}$ The reason for the probability of provision being zero in any equilibrium in this open ascending auction mechanism is the following. Because of the sequential nature of the contributions, each bidder is eager to be the first one to contribute in order to free ride on the contribution of the other bidder. By committing to a low level of contribution, a bidder can force the other to contribute the rest of the cost. Therefore no bidder is willing to win the auction; that is, no bidder wants to be the last one to drop out. Hence the bids are too low and as a result there is no provision. This is particularly striking, because although the first bidder to drop out is able to free ride on the other bidder, he also faces the risk that the other bidder may not value the public good enough for it to be provided.

We also consider a natural variation of the open ascending auction described above and check if our result still holds. In this variation, the auction stops once the first bidder drops out and the other bidder simply decides whether to pay the rest of the cost or not. The difference between the two auctions is that in the original format the second bidder to drop out has to pledge an amount at least as big as the drop out price of the first bidder. In the variation, the second bidder is not constrained by the bid of the other bidder from below and hence can pledge any amount he wants. The reason we consider this variation is the following. In the original mechanism, bidders are reluctant to wait until the price reaches a high level, because if there is provision, the second bidder to drop out has to pay his drop out price which might be larger than his value. However, such a possibility of a negative payoff is not present in the variation described. A bidder can always choose not to contribute the rest if he is not the first to drop out, thus avoiding the possibility of a negative payoff. Therefore, the bidders may wait longer to drop out in this variation than they would wait in the original mechanism. However, we show that our result still holds; that is, there is no provision of the public good in this variation.

The alternative mechanism we propose in the light of this strong inefficiency of the open ascending auction works as follows. First, the right to contribute first is auctioned off through a second price sealed-bid auction. The winner pays the loser's bid and earns the right to contribute first. Then, bidders play a sequential contribution game with the order determined in the auction. The idea

\footnotetext{
${ }^{3}$ With a full refund feature, contributors are not worried about potential negative payoffs. Hence they tend to contribute more relative to the no refund case.
} 
behind this mechanism is the following. We have seen that the bidders value being the first one to contribute and hence to get a chance to free ride on the other bidder's contribution. Since what is being auctioned off should be valuable to the bidders, we auction off the right to contribute first. This way the winner pays a price in order to free ride on the other bidder's contribution. We show that our mechanism under weak conditions, weakly outperforms the sequential contribution mechanism with exogenous order, the benchmark mechanism, in terms of the probability of provision. That is, the benchmark mechanism never generates a probability of provision that is strictly higher than the probability of provision in our mechanism. This is because in our mechanism, if there is no payment and no information revelation, the bidders play the sequential contribution game with the same strategies as in the benchmark mechanism. If some information is revealed to the winner, then the probability of provision can only weakly increase. We also provide an example where our mechanism generates a strictly higher probability of provision than the benchmark does.

This paper is related to a number of other papers in the literature. Varian (1994) considers a sequential contribution game under complete information and argues that the ability of the first mover to credibly commit to a certain level of contribution aggravates the free rider problem. Bag and Roy (2008), on the other hand, show that under incomplete information about individual private valuations for the public good, a sequential contribution mechanism may perform better than the simultaneous contribution game, in terms of total expected contributions. Both papers work with continuous public goods. Alboth, Lerner, and Shalev (2001) and Menezes, Monteiro and Temimi (2001) use tools familiar from auction theory and study continuous equilibria in the private provision game. Barbieri and Malueg (2008) consider a profit maximizing seller with a degree of altruism and restrict analysis to piecewise-linear equilibria. They show that the seller chooses the "subscription game," a simultaneous contribution game with full refunds as the optimal provision mechanism within the restricted domain. Deb and Razzolini (1999) use auction-like mechanisms to allocate excludable public goods.

\section{The Model}

There are two bidders, $A$ and $B$. Each bidder $i$ assigns a value $x_{i}$, the realization of a random variable $X_{i} \in[0,1]$, to the public good. Each $X_{i}$ is independently and identically distributed on $[0,1]$ according to the cumulative distribution function $F(\cdot)$ which has full support. The associated density function is 
$f(\cdot)$. Bidders are risk neutral. Bidder $i$ knows only the realization $x_{i} \in X_{i}$ and that the other bidder's value is independently distributed according to $F(\cdot)$. The cost of providing the public good is given by a constant $c$ such that $1<c<2$. Note that no bidder is willing to provide the public good on his own. Also note that there are some combinations of valuations for which it is efficient to provide the good as well as other combinations for which it is not efficient to do so. All of the above is common knowledge to both bidders.

We will first consider an open ascending auction in which bidders pay their drop-out prices and these payments are contributed towards the provision of the public good. This is a natural way to think about dynamic auctions in the public good setting. Then, using the insights from the auction mechanism mentioned above, we will propose another mechanism in which the right to contribute first is auctioned off. The winner pays the other bidder's bid and gets to contribute first in the sequential contribution game following the auction. In both mechanisms, the payments are fully refunded if the public good is not provided, that is, if the total contribution is less than the cost of the public good.

\section{All-pay open ascending auction}

The rules of the auction are as follows. The auctioneer sets the price at zero and continuously raises it. Each bidder observes the ascending price and drops out at his preferred price. Bidders may drop out at any price, but once they do so, they cannot reenter the auction. The auction ends when there is no active bidder; that is, when both bidders have dropped out. When the auction is over, if the sum of the prices at which the bidders have dropped out is at least as big as the cost, $c$, the public good is provided and each bidder pays his own drop out price. If the sum of the drop out prices is more than $c$, the excess contribution is kept by the auctioneer. If the sum of the drop out prices is less than $c$, then the public good is not provided and no payments are made. If the bidders drop out at the same price, the auctioneer selects one of the bidders to be the first one to drop out by a fair coin toss. ${ }^{4,5}$ If the public good is provided, bidder $i$ gets a payoff $x_{i}-b$ where $b$ is his drop out price. If the good is not provided, each bidder receives zero.

Note that every bidder pays his own bid. If we let the bidder who drops out first pay nothing, then there is no hope for provision of the public good simply because no bidder provides the good on

\footnotetext{
${ }^{4}$ Since we will allow bidding functions that are constant over some range, we need a tie-breaking rule.

${ }^{5} \mathrm{~A}$ natural alternative assumption would be that if both bidders drop out at the same price, then the auction ends. We conjecture that our result carries over to this case.
} 
his own. Hence a natural adaptation of open ascending auctions to the context of a discrete public good provision has to have an all-pay element: we have to make the bidder who drops out first pay some amount, and a natural amount is his own bid.

A strategy for bidder $i$ is a pair denoted $\left(\beta_{i 1}, \beta_{i 2}\right)$, where $\beta_{i 1}:[0,1] \rightarrow \mathbb{R}_{+}$, and $\beta_{i 2}:[0,1] \times \mathbb{R}_{+} \rightarrow$ $\mathbb{R}_{+} \cdot \beta_{i 1}(x)$ is bidder $i$ 's drop out price given that the other bidder has not dropped out yet. $\beta_{i 2}\left(x, b_{1}\right)$ is his drop out price given that the other bidder has already dropped out at price $b_{1}$. So we require $\beta_{i 2}\left(x, b_{1}\right) \geq b_{1}$.

Our equilibrium concept is Perfect Bayesian Equilibrium, equilibrium henceforth. An equilibrium is a strategy profile $\left(\left(\beta_{A 1}, \beta_{A 2}\right),\left(\beta_{B 1}, \beta_{B 2}\right)\right)$ and beliefs $f\left(\cdot \mid b_{1}\right)$ such that at any stage of the game, strategies are optimal given the beliefs, and the beliefs are obtained from equilibrium strategies and observed actions using Bayes' rule whenever possible.

The bidder who drops out second, essentially chooses between staying active until the price reaches a level which is enough to cover the rest of the cost or dropping out before the price reaches that level. Therefore, in any equilibrium, $\beta_{i 2}(\cdot, \cdot)$ takes the following form

$$
\beta_{i 2}\left(x_{i}, b_{1}\right)=\left\{\begin{array}{ccc}
c-b_{1} & \text { if } & b_{1}<c / 2 \text { and } x_{i}-\left(c-b_{1}\right) \geq 0 \\
b \in\left[b_{1}, c-b_{1}\right) & \text { if } & b_{1}<c / 2 \text { and } x_{i}-\left(c-b_{1}\right)<0 \\
b_{1} & \text { if } & b_{1} \geq c / 2
\end{array}\right.
$$

Note that no payments are made in case the public good is not provided. So, if the first bidder's bid is not enough for the second bidder to be willing to cover the rest of the cost, the second bidder can drop out at any price $b$ less than $c-b_{1}$, so that the public good is not provided. If the the first bidder drops out at a price $b_{1}$ equal to or higher than $c / 2$, then there is provision regardless of the second bidder's drop out price. Thus, the second bidder drops out immediately at $b_{1}$ to minimize his payment.

Since the equilibrium bidding function, $\beta_{i 2}\left(x_{i}, b_{1}\right)$, for $i=A, B$, is already given above, we only need to focus on $\beta_{i 1}\left(x_{i}\right)$. So whenever we talk about an equilibrium, we will specify only $\beta_{i 1}\left(x_{i}\right)$, $i=A, B$, omitting $\beta_{i 2}\left(x_{i}, b_{1}\right)$ to save space. For ease of notation, we will drop the subscript 1 and write $\beta_{i}(\cdot)$. If $i=A(B)$, then $-i$ will denote bidder $B(A)$. Also, instead of $x_{i}$ and $x_{-i}$, we will use $x$ and $y$, respectively, to avoid the subscripts. We will let $\beta_{i}^{-1}$ denote $\beta_{i}$ 's inverse. In what follows, we will use the terms drop out price and bid interchangeably, as well as the terms contributor and bidder. Finally we will denote the expected payoff of bidder $i$ from bidding $b$ when his value is $x$ and bidder 
$-i$ bids according to $\beta_{-i}(\cdot)$ by $\pi^{i}\left(x, b, \beta_{-i}\right)$.

The payoff for bidder $i$ with value $x$ when he bids $b$ is given by

$$
\left\{\begin{array}{ccc}
x-\beta_{-i}(y) & \text { if } & b \geq \beta_{-i}(y) \geq c / 2 \\
\left(x-\left(c-\beta_{-i}(y)\right)\right) I_{x-\left(c-\beta_{-i}(y)\right) \geq 0} & \text { if } & b>\beta_{-i}(y) \text { and } \beta_{-i}(y)<c / 2 \\
x-b & \text { if } & c / 2 \leq b<\beta_{-i}(y) \\
x-b & \text { if } & b<\beta_{-i}(y), y+b>c \text { and } b<c / 2 \\
0 & \text { if } & b<\beta_{-i}(y), y+b<c \text { and } b<c / 2 \\
1 / 2\left(x-\left(c-\beta_{-i}(y)\right)\right) I_{x-\left(c-\beta_{-i}(y)\right) \geq 0}+1 / 2(x-b) I_{y+\beta_{i}(x) \geq c} & \text { if } & b=\beta_{-i}(y)<c / 2
\end{array}\right.
$$

where

$$
I_{A(x, y)}=\left\{\begin{array}{ccc}
1 & \text { if } & x, y \text { satisfy condition } A(x, y) \\
0 & \text { if } & \text { otherwise }
\end{array}\right.
$$

If bidder $i$ is the last one to drop out and the first bidder has dropped out at a price less than $c / 2$, then his payoff is zero if he chooses not to pay the rest of the cost and $x-\left(c-\beta_{-i}(y)\right)$ if he chooses to pay the rest. If bidder $i$ is the last one to drop out and the first bidder has dropped out at a price higher than $c / 2$, then he drops out immediately at the first bidder's drop out, thus there is provision and his payoff is $x-\beta_{-i}(y)$. If he is the first one to drop out, then he gets a payoff $x-b$ if the other bidder contributes the rest, and zero otherwise. ${ }^{6}$

Below we introduce a definition which simplifies the analysis.

Definition 1 Let $\mu_{\beta_{i}}$ be the probability measure over $\left\{\beta_{i}(x) \mid x \in[c-1,1]\right\}$. Then define $\bar{\beta}_{i}=\max$ $\operatorname{supp}\left(\mu_{\beta_{i}}\right)$, where $\operatorname{supp}\left(\mu_{\beta_{i}}\right)$ is the support of $\mu_{\beta_{i}}$.

Consider an equilibrium, $\left(\beta_{A}(\cdot), \beta_{B}(\cdot)\right)$, with $\bar{\beta}_{i} \leq c-1$ for some $i \in\{A, B\}$. In such an equilibrium, there is no provision. To see this, first note that if a bidder is bidding above $c-1$ when his value is less than $c-1$, then he must be the second to drop out almost everywhere. Otherwise he receives a negative payoff when he is the first one to drop out and zero when he is the second to drop out. Thus, he deviates to a bid below $c-1$ and get zero payoff. Now, if $\bar{\beta}_{i} \leq c-1$, then bidder $-i$, if he is the second to drop out, will not pay the rest of the cost since valuations are at most 1 . If bidder $-i$ is the first one to drop out, then his bid must be less than $c-1$. Thus, bidder $i$ will not pay the rest of the

\footnotetext{
${ }^{6}$ If bidder $i$ is the first to drop out with a drop out price $b$ less than $c / 2$, then it is irrelevant whether the other bidder has a value $y$ such that $y+b=c$. This is because such an event has probability zero. Thus, we omit the case $y+b=c$.
} 
cost. Thus, there is no provision of the public good and both bidders get zero payoff. We call such an equilibrium a trivial equilibrium as stated in the following definition.

Definition $2 A$ trivial equilibrium is an equilibrium, $\left(\beta_{A}(\cdot), \beta_{B}(\cdot)\right)$, such that $\bar{\beta}_{i} \leq c-1$ for some $i \in\{A, B\}$.

A trivial equilibrium always exists. Any $\left(\beta_{A}(\cdot), \beta_{B}(\cdot)\right)$, such that $\beta_{i}(x) \leq c-1$ for all $x$, for both $i=A, B$ is a trivial equilibrium. To see this, first note that both bidders get zero payoff since there is no provision of the public good. Given that bidder $i$ uses a strategy $\beta_{i}(x)$ such that $\beta_{i}(x) \leq c-1$ for all $x$, bidder $-i$ has no profitable deviation. This is because the good will never be provided even if bidder $-i$ drops out at a price higher than $c-1$ for some positive measure set of values.

Now we will study the set of equilibria of the auction described above. In particular, we will show that the probability of provision is zero in any equilibrium, through a series of lemmas. The first lemma says that, given that the other bidder has not dropped out yet, bidder $i$ will drop out before the price exceeds half of the cost.

Lemma 1 Consider a non-trivial equilibrium, $\left(\beta_{A}(\cdot), \beta_{B}(\cdot)\right)$. Then $\beta_{i}(x) \leq c / 2$ for all $x \in[0,1]$, for $i=A, B$.

Proof. If no one has dropped out yet when the price hits $c / 2$, it is optimal to drop out immediately at $c / 2$. This is because the other bidder will have to pay at least $c / 2$, since bidders pay their drop out price in case the good is provided. Therefore, bidders will wait at most until the price hits $c / 2$.

Note that the price at which a bidder drops out given that the other bidder has already dropped out can be above $c / 2$.

To show that there exists no non-trivial equilibrium, we will first focus on equilibrium bidding functions which are non-decreasing. The following lemma below shows that in a non-trivial equilibrium with non-decreasing bidding functions both bidders receive a positive expected payoff for large enough valuations.

Lemma 2 Consider a non-trivial equilibrium, $\left(\beta_{A}(\cdot), \beta_{B}(\cdot)\right)$, with non-decreasing bidding functions. Then $\pi^{i}\left(x, \beta_{i}(x), \beta_{-i}\right)>0$ for all $x \in(c-1,1]$, for $i=A, B$. 
Proof. Suppose $\left(\beta_{A}(\cdot), \beta_{B}(\cdot)\right)$ is a non-trivial equilibrium. By definition $\bar{\beta}_{i}>c-1$ for $i=A, B$. Suppose that bidder $i$ with a value $x \in(c-1,1]$ receives a zero expected payoff, $\pi^{i}\left(x, \beta_{i}(x)\right)=0$. But then bidder $i$ could deviate to a bid $c-1+\varepsilon$, for some small $\varepsilon>0$ such that $c-1+\varepsilon<\min \left\{x_{i}, \bar{\beta}_{-i}\right\}$ and get a positive expected payoff. To see this, note that the fact that this bid is strictly below $\bar{\beta}_{-i}$ and the fact that $\beta_{-i}$ is non-decreasing implies that there is a positive measure set of values of bidder $-i$, say $[1-\delta, 1]$, such that bidder $-i$ drops out second when $i$ makes this bid. Since $-i$ would pay the rest of the cost whenever his type is in $(1-\varepsilon, 1]$, we see that the public good is provided and $i$ 's payoff is strictly positive when $-i$ 's value is in $(\max \{1-\delta, 1-\varepsilon\}, 1]$, a set with strictly positive measure. Since an equilibrium expected payoff can never be strictly smaller than the expected profit from a deviation, the result follows.

The next lemma says that in the relevant range, no bidder bids more than his valuation for the public good in a non-trivial equilibrium with non-decreasing bidding functions

Lemma 3 Consider a non-trivial equilibrium, $\left(\beta_{A}(\cdot), \beta_{B}(\cdot)\right)$, with non-decreasing bidding functions. Then $\beta_{i}(x) \leq x$ for all $x \in(c-1,1]$, for $i=A, B$.

Proof. Suppose $\beta_{i}(\widehat{x})>\widehat{x}$ for some $\widehat{x} \in(c-1,1]$. Suppose the price has just hit his value $\widehat{x}$. If bidder $-i$ drops out at this instant, then bidder $i$ 's payoff is zero. To see this, note that bidder $-i$ pays $\widehat{x}$ and bidder $i$ pays at most $\widehat{x}$, making at most $2 \widehat{x}$ in total. For it to be possible for bidder $i$ to bid above $\widehat{x}$, we must have $\widehat{x}<c / 2$, so $2 \widehat{x}<c$. Hence the public good is not provided. If bidder $-i$ does not drop out at $\widehat{x}$, then bidder $i$ 's expected payoff is strictly negative unless the probability of provision is zero. To see this, note that if the public good gets provided, then bidder $i$ will have to pay at least the lower of the two drop out prices. If he is the first to drop out, he pays his bid which is above $\widehat{x}$. If he is the second to drop out he pays the rest of the cost which is at least $c / 2$ thus strictly bigger than $\widehat{x}$. Hence when the price reaches $\widehat{x}$, bidder $i$ will only keep being active if the probability of provision is zero conditional on price reaching $\widehat{x}$. Thus, his expected payoff is zero. But we know from Lemma 2 that this cannot be the case in a non-trivial equilibrium with non-decreasing bidding functions.

When $\beta_{i}(x)$ is non-decreasing almost everywhere over the range $(c-1,1]$, define $x^{i}$ to be the value such that $\beta_{i}(x) \geq c-x^{i}$ almost everywhere over the range $\left(x^{i}, 1\right]$, and $\beta_{i}(x) \leq c-x^{i}$ almost everywhere over the range $\left(c-1, x^{i}\right) \cdot{ }^{7}$ Let $b^{i}=c-x^{i} \cdot y^{-i}$ and $b^{-i}$ are defined analogously.

\footnotetext{
${ }^{7}$ If $\beta_{i}(x) \leq c-1$ for almost all $x$, then $x^{i}=1$. If $\beta_{i}(x) \geq c$ for almost all $x$, then $x^{i}=0$. In any other case, there
} 
Lemma 4 Consider a non-trivial equilibrium, $\left(\beta_{A}(\cdot), \beta_{B}(\cdot)\right)$, with bidding functions non-decreasing almost everywhere over the range $(c-1,1]$. Then $\beta_{i}(x) \leq b^{-i}$, for almost all $x \in(c-1,1]$, for $i=A, B$.

Proof. See Appendix A.

The intuition for Lemma 4 is as follows. Suppose bidder $i$ has a value $x$ less than $y^{-i}$, that is, $c-x>b^{-i}$. Suppose bidder $i$ 's bid $b$ is such that $c-x>b>b^{-i}$. If he is the first to drop out, then he gets a payoff of $x-b>0$ since $\beta_{i}(y) \geq b>b^{-i}$ implies $y$ is such that $y>c-b$. If bidder $i$ is the second to drop out, then his payoff is zero as $c-x>b \geq \beta_{i}(y)$ implies $x+\beta_{-i}(y)<c$. As bidder $i$ lowers his bid, the range in which he is the first to drop out expands. Thus he receives a higher payoff over a bigger range. Now suppose that bidder $i$ 's bid $b$ is such that $b>c-x>b^{-i}$. If he is the first to drop out, he gets a payoff $x-b$ since $\beta_{i}(y) \geq b>b^{-i}$ implies $y$ is such that $y>c-b$. If he is the second to drop out, then there is a range over which he gets $x-c+\beta_{-i}(y)$ since $b \geq \beta_{i}(y)>c-x$ over some range which implies $x>c-\beta_{i}(y)$. If he lowers his bid by $\varepsilon$, the range over which he gets $x-b+\varepsilon$ is bigger than the range over which he would get $x-b$, and the range where he gets $x-c+\beta_{-i}(y)$ is smaller. Since $b \leq c / 2$ by Lemma 1 , we have $x-b>x-c+\beta_{-i}(y)$. Thus, bidder $i$ lowers his bid $b$ whenever $b>b^{-i}$. A similar argument applies if bidder $i$ has a value $x$ bigger than $y^{-i}$. Thus, in a non-trivial equilibrium, no bidder $i$ bids above $b^{-i}$.

Lemma 5 There exists no non-trivial equilibrium, $\left(\beta_{A}(\cdot), \beta_{B}(\cdot)\right)$, with bidding functions that are nondecreasing almost everywhere over the range $(c-1,1]$.

Proof. See Appendix A.

To see the idea behind Lemma 5 , first note that Lemma 4 implies that $\beta_{i}(x)=b^{-i}$ for all $x>y^{i}$ for $i=A, B$, and hence $b^{i}=b^{-i}$. Then any type of bidder $i$ who bids $b^{i}$ will deviate by cutting his bid by a small enough amount. By bidding $b^{i}$, he is the first one to drop out with probability $1 / 2$ for the values $y \in\left[y^{i}, 1\right]$ of bidder $-i$. By deviating to $b^{i}-\varepsilon$, where $\varepsilon>0$ is arbitrarily small, he is the first one to drop out with probability 1 for all $y \in\left[y^{i}, 1\right]$. Note that the first bidder to drop out pays less than $c / 2$, while the other bidder pays more than $c / 2$, and over the range $\left[y^{i}, 1\right]$ bidder $i$ decreases the probability of provision by only a small amount by cutting his bid by $\varepsilon$. Hence the loss can be made arbitrarily small by taking $\varepsilon$ sufficiently small while the net gain is bounded above by zero for all $\varepsilon$.

exists a $x^{i} \in(0,1)$. To see this, first note that if there exist a value $x^{\prime}$ such that $\beta_{i}\left(x^{\prime}\right)=c-x^{\prime}$, then there is exactly one such $x^{\prime}$ and $x^{i}=x^{\prime}$ since $\beta_{i}$ is non-decreasing. If there is no such $x^{\prime}$, then there exists a unique value $x^{\prime \prime}$ such that $\beta_{i}(x)>c-x^{\prime \prime}$ for all $x>x^{\prime \prime}$, and $\beta_{i}(x)<c-x^{\prime \prime}$ for all $x<x^{\prime \prime}$, since $\beta_{i}$ is non-decreasing. Thus $x^{i}=x^{\prime \prime}$. 
Having showed that there is no non-trivial equilibrium with non-decreasing bidding functions, now we focus on bidding functions that are decreasing over some range.

Lemma 6 Consider a non-trivial equilibrium, $\left(\beta_{A}(\cdot), \beta_{B}(\cdot)\right)$. For any $x_{1}, x_{2} \in(c-1,1]$ such that $x_{1}<x_{2}$, if $\beta_{i}\left(x_{1}\right) \leq x_{1}$ and $\beta_{i}\left(x_{2}\right) \leq x_{2}$, then $\beta_{i}\left(x_{1}\right) \leq \beta_{i}\left(x_{2}\right)$, for $i=A, B$.

Proof. See Appendix A.

The next lemma shows that in a non-trivial equilibrium with a bidding function that is decreasing over some range, the probability of provision must be zero.

Lemma 7 In a non-trivial equilibrium, $\left(\beta_{A}(\cdot), \beta_{B}(\cdot)\right)$, the probability of provision is zero.

Proof. Fix a non-trivial equilibrium, $\left(\beta_{A}(\cdot), \beta_{B}(\cdot)\right)$. Suppose that the probability of provision is strictly positive in this equilibrium. If the probability of provision is strictly positive when bidder $i$ has a valuation $x$, then the probability of provision must be strictly positive when bidder $i$ has a valuation $x^{\prime}>x$. Otherwise, bidder $i$ 's payoff is zero when he has valuation $x^{\prime}$, but he can deviate to the bid of type $x$ and have a strictly positive expected payoff. Now define $x_{i}^{*}$ to be the infimum over the set of values of bidder $i$ such that the probability of provision in this equilibrium is positive. ${ }^{8}$ Then, the probability of provision is strictly positive for all $x \in\left(x_{i}^{*}, 1\right]$. Note that $x_{i}^{*} \geq c-1$, since in any equilibrium the probability of provision is zero when $x \leq c-1$. Since we assumed that the equilibrium we fixed has a strictly positive probability of provision, $x_{i}^{*}<1$. Using the argument in the proof of Lemma 2 together with the fact that the expected payoff is strictly positive for any $x \in\left(x_{i}^{*}, 1\right]$, we conclude that for any $x \in\left(x_{i}^{*}, 1\right], \beta_{i}(x) \leq x .{ }^{9}$ By Lemma $6, \beta_{i}(x)$ is non-decreasing over the range $\left(x_{i}^{*}, 1\right]$. Since probability of provision is zero for all $x \in\left[0, x_{i}^{*}\right]$, by a similar argument used in the proofs of Lemma 4 and Lemma 5, we can show that this cannot be a non-trivial equilibrium. Therefore the probability of provision cannot be strictly positive.

The following proposition sums up the results.

Proposition 1 In the all-pay open ascending auction, the probability of provision is always zero.

\footnotetext{
${ }^{8}$ Note that $x_{i}^{*}$ depends on the equilibrium. To save space we write $x_{i}^{*}$ instead of $x_{i}^{*}\left(\beta_{A}(\cdot), \beta_{B}(\cdot)\right)$.

${ }^{9}$ The argument in Lemma 2 uses the fact that the expected payoff is positive, which is ensured by having nondecreasing bidding functions. Here, however, even though we do not impose bidding functions to be non-decreasing, we know that for any $x \in\left(x^{*}, 1\right]$, the expected payoff is strictly positive. Hence the argument in Lemma 2 applies.
} 
The intuition for this result is as follows. First observe that in a sequential contribution game with exogenous order, being the first one to contribute is advantageous since the first player to contribute, by committing to a low level of contribution, can free ride, in expectation, on the other player's contribution. In the all-pay auction game, being the first to drop out is essentially being the first contributor and hence advantageous. Thus, for a bidder who is the second to drop out it will be profitable to undercut the other's bid to be the first one to contribute. This incentive to deviate disappears only when almost all bids are less than $c-1$ for values that are at least $c-1$, that is when the equilibrium is trivial.

\subsection{A Variation}

One natural variation of the mechanism discussed above is an auction procedure in which when the first bidder drops out, the auctioneer stops the auction and then lets the other bidder simply decide whether to pay the residual cost or not, given that the contribution of the bidder who dropped out is his drop out price.

To be more precise, the mechanism works as follows. The auctioneer, starting at zero price, continuously increases the price. Each bidder observes the ascending price and drops out at his preferred price. Bidders may drop out at any price, but once they do so, they cannot reenter the auction. If both bidders drop out at the same price, the auctioneer selects one of the bidders to be the first one to drop out by a fair coin toss. When the first bidder drops out, the auction ends and the price at which he drops out is his contribution. Then the other bidder, having observed the first bidder's contribution, chooses to contribute the rest or not. If the second bidder contributes the rest, then the public good is provided. If not, then the good is not provided and no payments are made. If the public good is provided, bidder $i$ gets a payoff of $x_{i}-b$ where $b$ is his contribution, and $x_{i}$ is his valuation for the good. If the good is not provided each bidder receives a payoff of zero.

Call this mechanism M. Mechanism M differs from the all-pay open ascending auction mechanism in the following way. In the all-pay open ascending auction, the second bidder to drop out has to pledge at least as much as the drop out price of the first bidder. However, in mechanism $\mathbf{M}$, the second bidder to drop out can pledge any amount he likes.

The following proposition shows that there is no non-trivial equilibrium in mechanism $\mathbf{M}$.

Proposition $\mathbf{2}$ In mechanism $\mathbf{M}$, the probability of provision is always zero. 
Proof. See Appendix B.

\section{Auctioning off the right to contribute first}

We have seen that a direct application of an open ascending auction format in a discrete public good setting severely fails: There is no equilibrium with positive probability of provision. The problem with such an auction mechanism is that the bidders do not value being the winner; that is, they do not value being the second one to drop out. Instead, they like to be the first one to drop out which enables them to credibly commit to a low contribution and hence free ride, in expected terms, on the other bidder's contribution. This suggests that we should be auctioning off what is considered to be valuable. Since being the first one to contribute is valuable for the bidders, we propose a mechanism in which the right to contribute first is auctioned off through a second-price sealed-bid auction. That is, the winner gets to be the first one to contribute in a sequential contribution game following the auction. Such a mechanism enables us to make the first contributor pay a price to be able to free ride on the other bidder's contribution.

We take the widely-studied sequential contribution mechanism with exogenous order as our benchmark mechanism. We show that for non-decreasing equilibria the mechanism where the right to contribute first is auctioned off, under weak conditions, weakly outperforms the benchmark mechanism, in terms of the probability of provision. ${ }^{10}$

The rules of the mechanism are as follows. In the first stage the right to contribute first is auctioned off through a second-price sealed bid auction. Each bidder simultaneously bids. The bidder with the highest bid wins and pays the other bidder's bid, $b_{1} \cdot{ }^{11}$ The payment made by the winner is used towards the provision of the public good. In case there is a tie in the auction, the winner is picked by a fair coin toss. In the second stage, the bidders play a sequential contribution game. First, the winner of the auction contributes an amount, $k_{1}$, on top of what he has already paid in the auction. So his total contribution is $b_{1}+k_{1}$. Then the loser of the auction contributes an amount, $k_{2}$, after observing the total contribution made by the winner, $b_{1}+k_{1}$. If the total contribution, $b_{1}+k_{1}+k_{2}$, is high enough to cover the cost, $c$, then the public good is provided. Otherwise, the good is not provided and no payments are made; that is, all payments and contributions are fully refunded. If the public

\footnotetext{
${ }^{10}$ Although we restrict attention to non-decreasing equilibria, we conjecture that all equilibria are non-decreasing.

${ }^{11}$ The winner observes $b_{1}$.
} 
good is provided, then a bidder gets a payoff of $x-b$ where $x$ is his valuation for the public good and $b$ is his total contribution, which is the sum of the contribution he made in the second stage and the price he paid in the auction in case he is the winner.

In what follows, we will call the mechanism described above the endogenous order mechanism and the sequential contribution mechanism with exogenous order the random order mechanism.

A strategy in the endogenous order mechanism for bidder $i$ is given by $\left(\beta_{i}, \kappa_{i 1}, \kappa_{i 2}\right)$, where $\beta_{i}$ : $[0,1] \rightarrow \mathbb{R}_{+}$, and $\kappa_{i 1}:[0,1] \times \mathbb{R}_{+} \times \mathbb{R}_{+} \rightarrow \mathbb{R}_{+}$. and $\kappa_{i 2}:[0,1] \times \mathbb{R}_{+} \times \mathbb{R}_{+} \times \mathbb{R}_{+} \rightarrow \mathbb{R}_{+} \cdot \beta_{i}(x)$ is bidder $i$ 's bidding strategy in the auction. Denote the price paid by the winner of the auction by $b_{1}$, and the bid of the winner by $b_{2}$, and the contribution made by the winner in the sequential contribution game by $k_{1}$. Then $\kappa_{i 1}\left(x, b_{1}, b_{2}\right)$ and $\kappa_{i 2}\left(x, b_{1}, b_{2}, k_{1}\right)$ are the contributions of bidder $i$, if he wins the auction and if he loses it, respectively.

An equilibrium is a strategy profile $\left(\left(\beta_{A}, \kappa_{A 1}, \kappa_{A 2}\right),\left(\beta_{B}, \kappa_{B 1}, \kappa_{B 2}\right)\right)$ and beliefs $f\left(\cdot \mid b_{1}\right)$ such that at any stage of the game, strategies are optimal given the beliefs, and the beliefs are obtained from equilibrium strategies and observed actions using Bayes' rule whenever possible. ${ }^{12}$

In the second stage, the loser of the auction will simply decide to pay the rest of the cost or not, given his valuation for the public good and the total contribution of the winner. This strategy is the same for both bidders, so we can drop the $i$ subscript. Hence $\kappa_{2}\left(x, b_{1}, b_{2}, k_{1}\right)$ is given by

$$
\kappa_{2}\left(x, b_{1}, b_{2}, k_{1}\right)=\left\{\begin{array}{cc}
c-b_{1}-k_{1} & \text { if } x-\left(c-b_{1}-k_{1}\right) \geq 0 \\
k \in\left[0, c-b_{1}-k_{1}\right) & \text { otherwise }
\end{array}\right.
$$

$\kappa_{i 1}\left(x, b_{1}, b_{2}\right)$ solves the following maximization problem

$$
\max _{k}\left(x-k-b_{1}\right)\left[1-F\left(c-b_{1}-k \mid b_{1}\right)\right]
$$

where $F\left(\cdot \mid b_{1}\right)$ is the cumulative distribution function associated with the posterior beliefs $f\left(\cdot \mid b_{1}\right)$. We impose the following assumption on the density function in order to ensure that the objective function in the above maximization problem is strictly concave on the equilibrium path.

Assumption $\frac{2 f(x)}{\left|f^{\prime}(x)\right|}>2-c$ for all $x \in[0,1]$.

An equilibrium is given by $\left(\left(\beta_{A}, \kappa_{A 1}, \kappa_{2}\right),\left(\beta_{B}, \kappa_{B 1}, \kappa_{2}\right)\right)$ where $\kappa_{A 1}, \kappa_{B 1}$ and $\kappa_{2}$ are characterized

\footnotetext{
${ }^{12}$ The equilibrium concept is Perfect Bayesian Equilibrium as in the previous sections.
} 
above.

The random order mechanism has a unique equilibrium in which both players adopt the strategy $\left(\tau_{1}(x), \tau_{2}\left(x, k_{1}\right)\right)$, where $\tau_{1}:[0,1] \rightarrow \mathbb{R}_{+}$and $\tau_{2}:[0,1] \times \mathbb{R}_{+} \rightarrow \mathbb{R}_{+}$are the contributions of the first and second contributor given that the first contributor has already contributed $k_{1}$, respectively. By the same argument used for $\kappa_{i 2}\left(x, b_{1}, k_{1}\right)$ above, $\tau_{2}(\cdot, \cdot)$ is given by

$$
\tau_{2}\left(x, k_{1}\right)=\left\{\begin{array}{cc}
c-k_{1} & \text { if } x-\left(c-k_{1}\right) \geq 0 \\
k \in\left[0, c-k_{1}\right) & \text { otherwise }
\end{array}\right.
$$

$\tau_{1}(x)$ solves the following maximization problem

$$
\max _{\tau}(x-\tau) \operatorname{Pr}(y \geq c-\tau)
$$

By the assumption above, the objective function is strictly concave and the solution is

$$
\tau_{1}(x)=x-\frac{1-F(c-\tau(x))}{f(c-\tau(x))}
$$

where $\tau(x) \geq 0 .{ }^{13}$ Denote the probability of provision generated in the equilibrium of the random order mechanism by $p^{*}$. The following proposition states that the random order mechanism can never generate a strictly higher probability of provision than the endogenous order mechanism does.

Proposition 3 Assume that $\frac{2 f}{\left|f^{\prime}\right|}>2-c>\frac{f}{\left|f^{\prime}\right|}$ and $f^{\prime}<0$. Then, for any non-decreasing and symmetric equilibrium of the endogenous order mechanism, the probability of provision is at least $p^{*}$.

Proof. Pick a non-decreasing and symmetric equilibrium of the endogenous order mechanism. Pick an arbitrary pair of valuations $x$ and $y$ such that $x+y>c \cdot{ }^{14}$ Denote the contribution function of the first contributor in the random order mechanism with $\tau(\cdot)$, and the contribution function of the winner of the auction in the endogenous order mechanism with $\kappa(\cdot)$, dropping $b_{1}$ and $b_{2}$ to save space.

Case 1: There is no provision in the random order mechanism regardless of the order. Then, clearly endogenous order mechanism cannot do worse, simply because the probability of provision cannot be negative.

\footnotetext{
${ }^{13}$ Otherwise, the left hand side of $\tau_{1}(x)=x-\frac{1-F(c-\tau(x))}{f(c-\tau(x))}$ is negative while the right hand side is $x \geq 0$.

${ }^{14}$ If $x+y<c$, then the probability of provision is zero in both mechanisms. We ignore $x+y=c$ because it is a measure zero event.
} 
Case 2: There is provision in the random order mechanism regardless of the order. Then there is also provision in the endogenous order mechanism. To see this, consider three subcases regarding possible information revelation in the endogenous order mechanism.

Case 2.1: The winner infers nothing about the valuation of the loser. Then, the winner, after paying $b_{1}$ (the bid of the loser) will contribute $\max \left\{\tau(x)-b_{1}, 0\right\}$ in the second stage; that is, his total contribution will be at least $\tau(x)$. So, if there is provision in the random order mechanism, there is also provision in the equilibrium of the endogenous order mechanism.

Case 2.2: The winner learns the exact value of the loser. Then the winner will contribute just as enough to make the loser pay the rest in the second stage. So, if there is provision in the random order mechanism, there is also provision in the equilibrium of the endogenous order mechanism.

Case 2.3: The winner infers that the valuation of the loser lies in a subinterval $[a, d]$ of $[0,1]$ where $0 \leq a<d \leq 1$. That is, the equilibrium bidding function of the loser is constant over the interval $[a, d]$. Since we are looking at the equilibrium path, Bayes' rule implies that the posterior is

$$
\widehat{f}=\left\{\begin{array}{cc}
z f(s) & \text { if } a \leq s \leq d \\
0 & \text { otherwise }
\end{array}\right.
$$

where $z=1 / \int_{a}^{d} f(s) d s>1$. Consider three subcases.

Case 2.3.1: Suppose that $c-\tau(x) \geq d$. Then, the probability of provision in the random order mechanism is zero. The probability of provision in the equilibrium of the endogenous order is positive since $c-\kappa(x) \leq d$.

Case 2.3.2: Suppose that $a<c-\tau(x)<d$. That is, $c-d<\tau(x)<c-a$. Note that $\tau(x)$ satisfies the first order condition

$$
(x-\tau(x)) f(c-\tau(x))-\int_{c-\tau(x)}^{1} f(s) d s=0
$$

Hence,

$$
\begin{aligned}
(x-\tau(x)) \widehat{f}(c-\tau(x))-\int_{c-\tau(x)}^{d} \widehat{f}(s) d s & =z\left\{(x-\tau(x)) f(c-\tau(x))-\int_{c-\tau(x)}^{d} f(s) d s\right\} \\
& =z \int_{d}^{1} f(s) d s \\
& \geq 0
\end{aligned}
$$


Then, the optimal $\kappa(x)$ is at least as big as $\tau(x)$. Thus if there is provision in the random order mechanism then there is also provision in the equilibrium of the endogenous order mechanism.

Case 2.3.3: Suppose that $c-\tau(x) \leq a$. That is, $c-d<c-a \leq \tau(x)$. Then for any $\kappa \in[c-d, c-a]$, we have $\kappa \leq \tau(x)$, so the strict concavity of the ex ante objective function implies

$$
(x-\kappa) f(c-\kappa)-\int_{c-\kappa}^{1} f(s) d s>0
$$

Hence,

$$
\begin{aligned}
(x-\kappa) \widehat{f}(c-\kappa)-\int_{c-\kappa}^{d} \widehat{f}(s) d s & =z\left\{(x-\kappa) f(c-\kappa)-\int_{c-\kappa}^{d} f(s) d s\right\} \\
& >z \int_{d}^{1} f(s) d s \\
& \geq 0
\end{aligned}
$$

Thus, the optimal $\kappa(x)$ is a corner solution, which is $\kappa(x)=c-a$. That is, $c-\kappa(x)=a$. Thus, there is provision in the endogenous order mechanism because $y \in[a, d]$ implies $y \geq c-\kappa(x)$.

Case 3: There is provision in the random order mechanism for one order but not the other. Without loss of generality, suppose there is provision when the bidder with valuation $x$ contributes first but not when the bidder with valuation $y$ contributes first. Thus the probability of provision in the random order mechanism given $(x, y)$ is $1 / 2$. We first prove that $x>y$. Let $\tau(z)$ be the contribution of the first player in the random order mechanism, characterized by

$$
(z-\tau(z)) f(c-\tau(z))=1-F(c-\tau(z))
$$

Define $G(z, \tau)=(z-\tau) f(c-\tau)-[1-F(c-\tau)]=0$. Then, by implicit function theorem

$$
\frac{d \tau}{d z}=-\frac{\partial G(z, \tau) / \partial z}{\partial G(z, \tau) / \partial \tau}=-\frac{f(c-\tau)}{\partial G(z, \tau) / \partial \tau}
$$

Note that $\partial G(z, \tau) / \partial \tau<0$ because it is the second derivative of the ex ante objective function which is strictly concave by the assumption $\frac{2 f}{\left|f^{\prime}\right|}>2-c$. If $|\partial G(z, \tau) / \partial \tau|<f(c-\tau)$, then $\frac{d \tau}{d z}>1$. That is, $\left|-f^{\prime}(c-\tau)(z-\tau)-2 f(c-\tau)\right|<f(c-\tau)$ implies $\frac{d \tau}{d z}>1$. Since $\partial G(z, \tau) / \partial \tau<0$, we have $\frac{d \tau}{d z}>1$ if $f^{\prime}(c-\tau)(z-\tau)+f(c-\tau)<0$; that is, $\frac{f(c-\tau)}{\left|f^{\prime}(c-\tau)\right|}<z-\tau$ since $f^{\prime}<0$. Since $2-c>\frac{f}{\left|f^{\prime}\right|}$ implies 
$\frac{f(c-\tau)}{\left|f^{\prime}(c-\tau)\right|}<z-\tau$, we get $\frac{d \tau}{d z}>1$. Since there is no provision when bidder with valuation $y$ moves first we have $x+\tau(y)<c$. Also since there is provision when bidder with valuation $x$ moves first, we have $y+\tau(x) \geq c$. Thus, $y+\tau(x)>x+\tau(y)$. That is, $\tau(x)-x>\tau(y)-y$ which implies $x>y$ since $\tau(z)-z$ is strictly increasing.

Since $x>y$ and the equilibrium is non-decreasing and symmetric, $\beta(x) \geq \beta(y)$. If $\beta(x)>\beta(y)$, then bidder with valuation $x$ wins and argument in case 2 applies, thus there is provision. If $\beta(x)=\beta(y)$, then the bidder with valuation $x$ wins with probability $1 / 2$. There is provision if he wins the auction; that is, the provision probability in the endogenous order mechanism given $(x, y)$ is $1 / 2$.

So we have shown that in all cases the probability of provision given $(x, y)$ in the endogenous order mechanism is at least the probability of provision given this $(x, y)$ in the random order mechanism.

Proposition 3 says that endogenous order mechanism weakly outperforms the random order mechanism in terms of provision probability, under weak conditions, when non-decreasing equilibria are concerned. Below we provide an example in which the endogenous order mechanism generates a strictly higher probability of provision than the random order mechanism does. Although the cumulative density function specified below does not have full support, we believe that this example illustrates our point.

Example. Suppose there are three valuations $x_{L}, x_{M}$ and $x_{H}$ such that $0<x_{L}<x_{M}<x_{H}<1$. Assume that $2 x_{L}<c$ and $x_{L}+x_{M}>c$. Suppose that the probabilities are given by $p\left(x_{s}\right)=p_{s}$ where $s \in\{L, M, H\}$ such that $0<p_{L}<p_{M}<p_{H}$. Assume that $p_{L}=\frac{c-2 x_{L}}{x_{M}-x_{L}} \cdot{ }^{15}$ We first show that $\left(\left(\beta_{A}, \kappa_{A 1}, \kappa_{2}\right),\left(\beta_{A}, \kappa_{A 1}, \kappa_{2}\right)\right)$ is an equilibrium where $\kappa_{1}$ and $\kappa_{2}$ are given above and $\beta_{i}(x)$, for $i=A, B$, is given as follows:

$$
\beta_{i}(x)=\left\{\begin{array}{ccc}
0 & \text { if } & x=x_{L} \\
c / 2 & \text { if } & x=x_{M} \text { or } x=x_{H}
\end{array}\right.
$$

Suppose also that the beliefs are such that any bid other than 0 and $c / 2$ by the loser will make the winner infer that the loser's valuation is $x_{H}$.

To check deviations, we start with the low type, $x_{L}$. Bidder $i$ with type $x_{L}$ will not deviate to $c / 2$. If he bids $c / 2$, then with probability $\left(1-p_{L}\right) \frac{1}{2}$ he will win the auction and pay $c / 2$ since there is provision so no refund is made. But $c / 2$ is strictly bigger than his value, $x_{L}$. With probability

\footnotetext{
${ }^{15}$ Since $x_{L}+x_{M}>c$ and $2 x_{L}<c$, we have $0<p_{L}<1$.
} 
$\left(1-p_{L}\right) \frac{1}{2}$ he will lose the auction and the other bidder, bidder $j$, will pay $c / 2$ and contribute no more in the second stage, because bidder $j$ thinks that bidder $i$ has value either $x_{M}$ or $x_{H}$. Hence $i$ 's payoff is zero in this case. With probability $p_{L}$, he will win the auction but he will infer that the bidder $j$ has value $x_{L}$, and hence there will be no point for bidder $i$ to contribute any positive amount. Thus bidder $i$ 's expected payoff is

$$
\left(1-p_{L}\right) \frac{1}{2}\left(x_{L}-c / 2\right)+\left(1-p_{L}\right) \frac{1}{2}(0)+p_{L}(0)<0
$$

Hence bidding $c / 2$ brings a negative expected payoff. However, bidding 0 yields a non-negative expected payoff. This is because, in case he loses the auction his payoff is non-negative and in case he wins the auction his payoff is zero since there is no provision. The low type will not deviate to any bid $b \in(0, c / 2)$. If he does so, there will be no gain if the other bidder's type is low. He makes sure he wins the auction against low types but there will be no provision even if he wins. Against the types $x_{M}$ and $x_{H}$, there is no gain either because the opponent will win the auction, paying less than $c / 2$, and will think that he has value $x_{H}$. Hence the winner will not contribute more, so the low type will not contribute the rest and his payoff will be zero. The low type will not deviate to any bid $b>c / 2$. This is true because if he bids higher than $c / 2$, he does not gain when the other bidder has low value, and has to pay $c / 2$ when the other bidder's value is either $x_{M}$ or $x_{H}$, just as for a deviation to $c / 2$.

Now we check deviations by the $x_{M}$ type. If bidder $i$ with type $x_{M}$ bids 0 , then with probability $p_{L} \frac{1}{2}$ he wins the auction and infers that the other bidder has type $x_{L}$. Hence he contributes $c-x_{L}$ and the other bidder contributes the rest. With probability $p_{L} \frac{1}{2}$ he loses the auction and bidder $j$ does not contribute any positive amount because bidder $j$ believes that bidder $i$ has value $x_{L}$. Thus there is no provision. With probability $\left(1-p_{L}\right)$, bidder $i$ loses the auction and bidder $j$ has value either $x_{M}$ or $x_{H}$. Bidder $j$ contributes $c-x_{L}$ because he believes bidder $i$ has value $x_{L}$. Thus the payoff for bidder $i$ is $x_{M}-\left(c-c+x_{L}\right)$. Therefore, the expected payoff for type $x_{M}$ from bidding 0 is given by

$$
\begin{aligned}
\pi\left(x_{M}, 0\right) & =p_{L} \frac{1}{2}\left(x_{M}-\left(c-x_{L}\right)\right)+p_{L} \frac{1}{2}(0)+\left(1-p_{L}\right)\left(x_{M}-\left(c-c+x_{L}\right)\right) \\
& =p_{L} \frac{1}{2}\left(x_{M}+x_{L}-c\right)+\left(1-p_{L}\right)\left(x_{M}-x_{L}\right)
\end{aligned}
$$


The expected payoff for type $x_{M}$ from bidding $c / 2$ is given by

$$
\begin{aligned}
\pi\left(x_{M}, c / 2\right) & =p_{L}\left(x_{M}-\left(c-x_{L}\right)\right)+\left(p_{M}+p_{H}\right) \frac{1}{2}\left(x_{M}-c / 2\right)+\left(p_{M}+p_{H}\right) \frac{1}{2}\left(x_{M}-c / 2\right) \\
& =p_{L}\left(x_{M}+x_{L}-c\right)+\left(1-p_{L}\right) \frac{1}{2}\left(x_{M}-c / 2\right)
\end{aligned}
$$

These two expected payoffs are the same when $p_{L}=\frac{c-2 x_{L}}{x_{M}-x_{L}}$. Therefore there is no incentive to deviate to 0 by the $x_{M}$ type. Now check the deviations to any $b \in(0, c / 2)$. There is no gain if the other bidder has type $x_{L}$. There is no gain if the other bidder has type $x_{M}$ or $x_{H}$ either, because he will pay $c / 2$ in any case, just like he does when he bids $c / 2$. Any deviation above $c / 2$ is not profitable either. Again there is no gain if the other bidder has type $x_{L}$. Also, there is no gain if the other bidder has value $x_{M}$ or $x_{H}$ either, again because he will pay $c / 2$ in each case.

Finally we check deviations by type $x_{H}$. Deviation to 0 is ruled out because $\pi\left(x_{H}, c / 2\right) \geq \pi\left(x_{H}, 0\right)$ when $p_{L} \geq \frac{c-2 x_{L}}{x_{H}-x_{L}}$. Since $x_{H}>x_{M}$, we have $p_{L}=\frac{c-2 x_{L}}{x_{M}-x_{L}}>\frac{c-2 x_{L}}{x_{H}-x_{L}}$. Any other deviation is not profitable by the same argument given for type $x_{M}$.

Now we specify $p_{M}, x_{s}$ and $s \in\{L, M, H\}$, and show that when the valuations are $x_{L}$ and $x_{M}$, there is provision in the equilibrium given above, but there is no provision in the random order mechanism. Suppose $x_{L}=c / 2-\varepsilon, x_{M}=c / 2+2 \varepsilon, x_{H}=c-\varepsilon$ and $p_{M}<\frac{1}{3} \frac{c-6 \varepsilon}{c-4 \varepsilon}$. Note that the conditions $2 x_{L}<c / 2$ and $x_{L}+x_{M}>c$ are satisfied with these specific values. Also note that, $p_{L}=\frac{c-2 x_{L}}{x_{M}-x_{L}}=\frac{2}{3}$. In the equilibrium described above, the $x_{M}$ type wins the auction, pays 0 , and infers that the loser has value $x_{L}$. Then in the second stage he contributes $c-x_{L}$, and there is provision. In the random order mechanism, however, there is no provision when the values are $x_{L}$ and $x_{M}$ and the former is the first one to contribute. To see this, first note that, the $x_{L}$ type will contribute either $c-x_{M}$ or $c-x_{H}$. He will contribute $c-x_{H}$ if

$$
\left(1-p_{L}-p_{M}\right)\left(x_{L}-\left(c-x_{H}\right)\right)>\left(1-p_{L}\right)\left(x_{L}-\left(c-x_{M}\right)\right)
$$

that is,

$$
\left(1-p_{L}\right)\left(x_{H}-x_{M}\right)>p_{M}\left(x_{L}+x_{H}-c\right)
$$

Plugging in the values, and arranging we get

$$
\frac{1}{3} \frac{c-6 \varepsilon}{c-4 \varepsilon}>p_{M}
$$


Therefore, type $x_{L}$ will contribute only $c-x_{H}$, and the good will not be provided since type $x_{M}$ will not pay the rest which is $c-\left(c-x_{H}\right)=x_{H}$.

\section{Conclusion}

In this paper, we explored the private provision of a discrete public good using auction mechanisms. A natural open ascending auction severely failed. No bidder wanted to win the auction, so the drop out prices were very low, leading to no provision at all. We then proposed an alternative mechanism in which the right to contribute first was auctioned off, followed by a sequential contribution game. This mechanism not only made the winner pay in order to free ride, but also endogenized the order. We showed that this mechanism performed weakly better than the sequential contribution mechanism with exogenous order.

\section{Appendix A}

Proof of Lemma 4. First, we consider the values of bidder $i$ such that $x<y^{-i}=c-b^{-i}$. That is, $c-x>b^{-i}$. We will consider the values $x>y^{-i}$ later. For now, suppose that $\beta_{-i}($.$) is strictly$ increasing. We will come back to the case where it is weakly increasing later. Suppose bidder $i$ bids an amount $b \geq b^{-i}$ when his value is $x$. There are two cases, $c-x \geq b \geq b^{j}$ and $b \geq c-x$.

(1) $c-x>b \geq b^{-i}$ : For any $y$ such that $y<\beta_{-i}^{-1}(b)$, bidder $-i$ drops out first and his drop out price is less than $c-x$. Hence for such values of bidder $-i$, there is no provision. For values $y$ such that $y>\beta_{-i}^{-1}(b)$, bidder $i$ drops out first and his drop out price, $b$, is bigger than $c-y$. Hence there is provision and bidder $i$ gets $x-b$ for such values of bidder $-i$. So the payoff for bidder $i$ is

$$
\pi^{i}\left(x, b, \beta_{-i}\right)=\int_{\beta_{-i}^{-1}(b)}^{1}[x-b] f(y) d y=(x-b)\left[1-F\left(\beta_{-i}^{-1}(b)\right)\right]
$$

As $b$ decreases, $(x-b)$ increases as well as $\left[1-F\left(\beta_{-i}^{-1}(b)\right)\right]$. Therefore in the range $c-x>b \geq b^{-i}$, optimal bid is $b^{-i}$.

(2) $b \geq c-x>b^{-i}:$ In this case, for values $y$, such that $y<\beta_{-i}^{-1}(c-x)$ there is no provision since bidder $-i$ drops out first and his drop out price is less than $c-x$. For values $y$ such that $\beta_{-i}^{-1}(c-x)<y<\beta_{-i}^{-1}(b)$, bidder $-i$ is still the first one to drop out, and his drop out price determines 
whether bidder $i$ will contribute the rest or not. For values $y$ such that $y>\beta_{-i}^{-1}(b)$, there is provision as explained above. So the payoff for bidder $i$ is given by

$$
\pi^{i}\left(x, b, \beta_{-i}\right)=\int_{\beta_{-i}^{-1}(c-x)}^{\beta_{-i}^{-1}(b)}\left[x-c+\beta_{-i}(y)\right] f(y) d y+(x-b)\left[1-F\left(\beta_{-i}^{-1}(b)\right)\right]
$$

Suppose instead of $b$, bidder $i$ bids $b-\varepsilon$, where $\varepsilon>0$. Then the relevant payoff is given by

$$
\pi^{i}\left(x, b-\varepsilon, \beta_{-i}\right)=\int_{\beta_{-i}^{-1}(c-x)}^{\beta_{-i}^{-1}(b-\varepsilon)}\left[x-c+\beta_{-i}(y)\right] f(y) d y+(x-b+\varepsilon)\left[1-F\left(\beta_{-i}^{-1}(b-\varepsilon)\right)\right]
$$

Then

$$
\begin{aligned}
\pi^{i}\left(x, b-\varepsilon, \beta_{-i}\right)-\pi^{i}\left(x, b, \beta_{-i}\right)= & (x-b+\varepsilon)\left[1-F\left(\beta_{-i}^{-1}(b-\varepsilon)\right)\right]-(x-b)\left[1-F\left(\beta_{-i}^{-1}(b)\right)\right] \\
& +\int_{\beta_{-i}^{-1}(c-x)}^{\beta_{-i}^{-1}(b-\varepsilon)}\left[x-c+\beta_{-i}(y)\right] f(y) d y-\int_{\beta_{-i}^{-1}(c-x)}^{\beta_{-i}^{-1}(b)}\left[x-c+\beta_{-i}(y)\right] f(y) d y \\
= & (x-b)\left[F\left(\beta_{-i}^{-1}(b)\right)-F\left(\beta_{-i}^{-1}(b-\varepsilon)\right)\right] \\
& +\varepsilon\left[1-F\left(\beta_{-i}^{-1}(b-\varepsilon)\right)\right]-\int_{\beta_{-i}^{-1}(b-\varepsilon)}^{\beta_{-i}^{-1}(b)}\left[x-c+\beta_{-i}(y)\right] f(y) d y \\
\geq & (x-b)\left[F\left(\beta_{-i}^{-1}(b)\right)-F\left(\beta_{-i}^{-1}(b-\varepsilon)\right)\right] \\
& +\varepsilon\left[1-F\left(\beta_{-i}^{-1}(b-\varepsilon)\right)\right]-\int_{\beta_{-i}^{-1}(b-\varepsilon)}^{\beta_{-i}^{-1}(b)}\left[x-c+\beta_{-i}\left(\beta_{-i}^{-1}(b)\right)\right] f(y) d y \\
= & (x-b)\left[F\left(\beta_{-i}^{-1}(b)\right)-F\left(\beta_{-i}^{-1}(b-\varepsilon)\right)\right] \\
& +\varepsilon\left[1-F\left(\beta_{-i}^{-1}(b-\varepsilon)\right)\right]-(x-c+b)\left[F\left(\beta_{-i}^{-1}(b)\right)-F\left(\beta_{-i}^{-1}(b-\varepsilon)\right)\right] \\
= & (c-2 b)\left[F\left(\beta_{-i}^{-1}(b)\right)-F\left(\beta_{-i}^{-1}(b-\varepsilon)\right)\right]+\varepsilon\left[1-F\left(\beta_{-i}^{-1}(b-\varepsilon)\right)\right] \\
> & 0
\end{aligned}
$$

The strict inequality follows from the fact that $b \leq c / 2$ by Lemma 1 , and that $\varepsilon>0$.

Therefore, the expected profit is strictly decreasing in $b$ over the range $\left[b^{-i}, \bar{\beta}_{i}\right)$. Hence whenever bidder $i$ has a value $x<y^{-i}$ and $\beta_{-i}($.$) is strictly increasing, he will never pick any bid higher than$ $b^{-i}$.

Now consider the case where $\beta_{-i}(y)=b$ for $y \in\left(y_{1}, y_{2}\right) \subseteq\left[y^{-i}, 1\right]$, for some $y_{1}, y_{2} \cdot{ }^{16}$ Again we consider two possible cases separately.

(1) $c-x>b \geq b-i$ : Over the range $\left(y_{1}, y_{2}\right)$ both bidders have the same bid, so with probability

\footnotetext{
${ }^{16}$ Since $b>b^{-i}$, we do not need to consider the case where bids coincide for values $y<y^{-i}$.
} 
$1 / 2$ bidder $i$ gets to be the first one to drop out and gets a payoff of $\int_{y_{1}}^{y_{2}}[x-b] f(y) d y$, and with probability $1 / 2$ he gets to be the last one to drop out and gets a payoff of zero since $b<c-x$. Hence the payoff when he bids $b$ when he has value $x$ is

$$
\pi^{i}\left(x, b, \beta_{-i}\right)=\frac{1}{2} \int_{y_{1}}^{y_{2}}[x-b] f(y) d y+\int_{y_{2}}^{1}[x-b] f(y) d y
$$

However if he bids $b-\varepsilon$, for an arbitrarily small $\varepsilon>0$, then his payoff is

$$
\pi^{i}\left(x, b-\varepsilon, \beta_{-i}\right)=\int_{\widetilde{y}_{1}}^{y_{2}}[x-b+\varepsilon] f(y) d y+\int_{y_{2}}^{1}[x-b+\varepsilon] f(y) d y
$$

where $\widetilde{y}_{1}$ is such that $\beta^{-i}\left(\widetilde{y}_{1}\right)=b-\varepsilon$ if $\beta_{-i}($.$) is continuous at y_{1}$, and $\widetilde{y}_{1}=y_{1}$ if $\beta_{-i}($.$) is not continuous$ at $y_{1}$. For $\varepsilon>0$ small enough, $\pi^{i}\left(x, b-\varepsilon, \beta_{-i}\right)$ is strictly bigger than $\pi^{i}\left(x, b, \beta_{-i}\right)$. Since any $b$ such that $b>b^{-i}$ is strictly dominated by $b-\varepsilon$, any bid $b \in\left(c-x, b^{-i}\right)$ cannot be optimal. Therefore, bidder $i$ bids $b^{-i}$.

(2) $b \geq c-x$ : Define $y_{0}$ such that $\beta_{-i}\left(y_{0}\right)=c-x \cdot{ }^{17}$ Then, bidder $i$ 's payoff when he has value $x$ and bids $b$ is given by

$$
\begin{aligned}
\pi^{i}\left(x, b, \beta_{-i}\right)= & \int_{y_{0}}^{y_{1}}\left[x-c+\beta_{-i}(y)\right] f(y) d y+\frac{1}{2} \int_{y_{1}}^{y_{2}}[x-b] f(y) d y+\frac{1}{2} \int_{y_{1}}^{y_{2}}[x-c+b] f(y) d y \\
& +\int_{y_{2}}^{1}[x-b] f(y) d y \\
= & \int_{y_{0}}^{y_{1}}\left[x-c+\beta_{-i}(y)\right] f(y) d y+\int_{y_{1}}^{y_{2}}[x-c / 2] f(y) d y+\int_{y_{2}}^{1}[x-b] f(y) d y
\end{aligned}
$$

However, the bidder with value $x$ can bid $b-\varepsilon$ and improve his payoff. To see this, let's first write the payoff from bidding $b-\varepsilon$.

$$
\pi^{i}\left(x, b-\varepsilon, \beta_{-i}\right)=\int_{y_{0}}^{\widetilde{y}_{1}}\left[x-c+\beta_{-i}(y)\right] f(y) d y+\int_{\widetilde{y}_{1}}^{y_{2}}[x-b+\varepsilon] f(y) d y+\int_{y_{2}}^{1}[x-b+\varepsilon] f(y) d y
$$

where $\widetilde{y}_{1}$ is defined as in case 1 above.

\footnotetext{
${ }^{17}$ If there is more than one such $y_{0}$, then pick the smallest one. Also note that, the existence of $y_{0}$ is not guaranteed since $\beta_{-i}($.$) is allowed to be discontinous. Even so, we can define y_{0}$ to be the value at which $\beta_{-i}($.$) has a jump with the$ property that $\beta_{-i}(y)<c-x$ for all $y<y_{0}$, and $\beta_{-i}(y)>c-x$ for all $y>y_{0}$.
} 
If $\widetilde{y}_{1}=y_{1}$, then $\pi^{i}\left(x, b-\varepsilon, \beta_{-i}\right)>\pi^{i}\left(x, b, \beta_{-i}\right)$ since

$$
\begin{aligned}
\int_{\widetilde{y}_{1}}^{y_{2}}[x-b+\varepsilon] f(y) d y & >\int_{y_{1}}^{y_{2}}[x-b] f(y) d y \\
& \geq \int_{y_{1}}^{y_{2}}[x-c / 2] f(y) d y
\end{aligned}
$$

where the last inequality follows from $b \leq c / 2$.

If $\widetilde{y}_{1}<y_{1}$, then $\pi^{i}\left(x, b-\varepsilon, \beta_{-i}\right)>\pi^{i}\left(x, b, \beta_{-i}\right)$ since

$$
\begin{aligned}
& \int_{y_{0}}^{\widetilde{y}_{1}}\left[x-c+\beta_{-i}(y)\right] f(y) d y+\int_{\widetilde{y}_{1}}^{y_{2}}[x-b+\varepsilon] f(y) d y \\
> & \int_{y_{0}}^{\widetilde{y}_{1}}\left[x-c+\beta_{-i}(y)\right] f(y) d y+\int_{\widetilde{y}_{1}}^{y_{2}}[x-c / 2] f(y) d y \\
> & \int_{y_{0}}^{y_{1}}\left[x-c+\beta_{-i}(y)\right] f(y) d y+\int_{y_{1}}^{y_{2}}[x-c / 2] f(y) d y
\end{aligned}
$$

where the first inequality follows from the fact that $x-b+\varepsilon>x-c / 2$, and the second inequality follows from the fact that $x-c / 2 \geq x-c+\beta_{-i}(y)$ and $\widetilde{y}_{1}<y_{1}$. Therefore any bid $b \geq c-x$ is strictly dominated by $b-\varepsilon$. Specifically $c-x$ will be dominated by $c-x-\varepsilon$. Hence we are back in case 1 , and optimal bid is $b^{-i}$.

Now consider the values $x>y^{-i}$. However in this case, there is only one region to check, which is $b>b^{-i}$, since $c-x<b^{-i}$. By the same argument in case 2 above we can conclude that $\pi^{i}\left(x, b-\varepsilon, \beta_{-i}\right)>$ $\pi^{i}\left(x, b, \beta_{-i}\right)$, and hence any $b>b^{-i}$ is dominated by $b^{-i}$.

Proof of Lemma 5. First note that it is straightforward from Lemma 4 that $b^{i}=b^{-i}$. Therefore we have $\beta_{i}(x) \leq b^{i}$ for all $x \in[0,1]$. Since $\beta_{i}($.$) is non-decreasing, definition of b^{i}$ implies that $\beta_{i}(x)=b^{i}$ for any $x>c-b^{i}=y^{i}$. So we have shown that $\beta_{i}(x)=\beta_{-i}(y)=b$ for $x, y \in[c-b, 1]$, in a non-trivial equilibrium with non-decreasing bidding functions (In fact, we have not shown that $\beta_{i}(c-b)=\beta_{-i}(c-b)=b$. However, if $\beta_{i}(c-b)<b$ and $\beta_{-i}(c-b)<b$, then one of the bidders deviates to $b-\varepsilon$, which will be clear below). We argue that this cannot be the case in a non-trivial equilibrium, simply because bidder $i$ would deviate to $b-\varepsilon$ for some $x$ where $\varepsilon>0$ is arbitrarily small. To see this, first note that bidder $i$ with value $x$ when he bids $b$, gets a payoff of $x-b$ with probability $1 / 2$ and a payoff of $x-c+b$ with probability $1 / 2$. Note also that $x-b \geq x-c+b$ since $b \leq c / 2$. However, dropping out at $b-\varepsilon$ generates a payoff of $x-b+\varepsilon$ over the range $[c-b+\varepsilon, 1]$, and zero 
over $[c-b, c-b+\varepsilon)$. So over the range $[c-b, 1]$, the net gain from deviating from $b$ to $b-\varepsilon$ is equal to

$$
\begin{aligned}
& \int_{c-b+\varepsilon}^{1}[x-b+\varepsilon] f(y) d y-\left\{1 / 2 \int_{c-b}^{1}[x-b] f(y) d y+1 / 2 \int_{c-b}^{1}[x-c+b] f(y) d y\right\} \\
= & {[x-b+\varepsilon][1-F(c-b+\varepsilon)]-\int_{c-b}^{1}[x-c / 2] f(y) d y } \\
= & {[x-b+\varepsilon][1-F(c-b+\varepsilon)]-[x-c / 2][1-F(c-b)] } \\
= & {[x-b][1-F(c-b+\varepsilon)]-[x-c / 2][1-F(c-b)]+\varepsilon[1-F(c-b+\varepsilon)] }
\end{aligned}
$$

This expression is positive for sufficiently small $\varepsilon>0$ since $x-b \geq x-c / 2{ }^{18}$ There is, however, a potential loss over the range $(\widetilde{y}, c-b)$ where $\widetilde{y}$ is defined to be the solution to $\beta_{-i}(y)=b-\varepsilon$ if there is a solution, and if there is no solution $\widetilde{y}$ is such that $\beta_{-i}(y)<b-\varepsilon$ for all $y<\widetilde{y}$, and $\beta_{-i}(y)>b-\varepsilon$ for all $c-b>y>\widetilde{y}$. This loss, however, depends on $\beta_{-i}(y)$ for $y<c-b$, and there are three possible cases. (1) $\beta_{-i}($.$) is not continuous at c-b$. Then $\beta_{-i}(y)<b$ for all $y<c-b$, which, together with discontinuity, implies $\widetilde{y}=c-b$. Hence the loss is zero. Therefore the deviation to $b-\varepsilon$ is profitable for any $x \in[c-b, 1]$. (2) $\beta_{-i}($.$) is continuous at c-b$, and $\beta_{-i}(y)<b$ for all $y<c-b$. Then the loss from deviation is equal to $\int_{\widetilde{y}}^{c-b}\left[x-c+\beta_{-i}(y)\right] f(y) d y$. However, for $x=c-b$, this expression is negative since $\beta_{-i}(y)<b$ for $y<c-b$. Hence the deviation to $b-\varepsilon$ is profitable for $x=c-b$. (3) $\beta_{-i}($.$) is continuous at c-b$, and $\beta_{-i}(y)=b$ for $y \in(\widehat{y}, c-b)$,for some $\widehat{y} \in[0, c-b)$. Then the loss is equal to $1 / 2 \int_{\widehat{y}}^{c-b}[x-c+b] f(y) d y$, which is zero when $x=c-b$. Hence, the deviation to $b-\varepsilon$ is profitable for $x=c-b$.

Proof of Lemma 6. Suppose that for bidder $i$ we have, $x_{1}<x_{2}$ and $\beta_{i}\left(x_{1}\right)>\beta_{i}\left(x_{2}\right)$, and bidder $-i$ bids according to $\beta_{-i}(y)$. We will show that the bidding function $\beta_{i}(x)$ is not incentive compatible. More precisely, we will show that $\pi^{i}\left(x_{2}, \beta_{i}\left(x_{2}\right), \beta_{-i}\right) \geq \pi^{i}\left(x_{2}, \beta_{i}\left(x_{1}\right), \beta_{-i}\right)$ implies $\pi^{i}\left(x_{1}, \beta_{i}\left(x_{2}\right), \beta_{-i}\right)>$ $\pi^{i}\left(x_{1}, \beta_{i}\left(x_{1}\right), \beta_{-i}\right)$, and hence we will conclude that such $\beta_{i}(x)$ cannot be decreasing over some set of

\footnotetext{
${ }^{18}$ If $b=c / 2$, the net gain over the range $[c / 2,1]$ is equal to

$$
\begin{aligned}
& {[x-c / 2][1-F(c / 2+\varepsilon)]-[x-c / 2][1-F(c / 2)]+\varepsilon[1-F(c / 2+\varepsilon)] } \\
= & {[F(c / 2)-F(c / 2+\varepsilon)][x-c / 2]+\varepsilon[1-F(c / 2+\varepsilon)] }
\end{aligned}
$$
}

which is positive for $x=c / 2$ and sufficiently small $\varepsilon>0$. 
values. To see this, first define

$$
\begin{aligned}
Y_{H} & =\left\{y \mid \beta_{-i}(y) \geq \beta_{i}\left(x_{1}\right)\right\} \\
Y_{M} & =\left\{y \mid \beta_{i}\left(x_{1}\right)>\beta_{-i}(y) \geq \beta_{i}\left(x_{2}\right)\right\} \\
Y_{L} & =\left\{y \mid \beta_{i}\left(x_{2}\right)>\beta_{-i}(y)\right\}
\end{aligned}
$$

There are five cases defined in terms of the relative magnitudes between $\beta_{i}\left(x_{k}\right)$ and $c-x_{k}$, where $k=1,2$.

(1) $c-x_{1}>c-x_{2}>\beta_{i}\left(x_{2}\right), c-x_{1}>\beta_{i}\left(x_{1}\right)$. The relevant payoffs are given as follows.

$$
\begin{aligned}
\pi^{i}\left(x_{1}, \beta_{i}\left(x_{1}\right), \beta_{-i}\right) & =\int_{Y_{H} \cap\left\{y \mid y \geq c-\beta_{i}\left(x_{1}\right)\right\}}\left[x_{1}-\beta_{i}\left(x_{1}\right)\right] f(y) d y=\left[x_{1}-\beta_{i}\left(x_{1}\right)\right] P_{1} \\
\pi^{i}\left(x_{2}, \beta_{i}\left(x_{2}\right), \beta_{-i}\right) & =\int_{\left(Y_{H} \cup Y_{M}\right) \cap\left\{y \mid y \geq c-\beta_{i}\left(x_{2}\right)\right\}}\left[x_{2}-\beta_{i}\left(x_{2}\right)\right] f(y) d y=\left[x_{2}-\beta_{i}\left(x_{2}\right)\right] P_{2} \\
\pi^{i}\left(x_{1}, \beta_{i}\left(x_{2}\right), \beta_{-i}\right) & =\int_{\left(Y_{H} \cup Y_{M}\right) \cap\left\{y \mid y \geq c-\beta_{i}\left(x_{2}\right)\right\}}\left[x_{1}-\beta_{i}\left(x_{2}\right)\right] f(y) d y=\left[x_{1}-\beta_{i}\left(x_{2}\right)\right] P_{2}
\end{aligned}
$$

$$
\begin{aligned}
\pi^{i}\left(x_{2}, \beta_{i}\left(x_{1}\right), \beta_{-i}\right) & =\int_{Y_{H} \cap\left\{y \mid y \geq c-\beta_{i}\left(x_{1}\right)\right\}}\left[x_{2}-\beta_{i}\left(x_{1}\right)\right] f(y) d y+\int_{Y_{M} \cap\left\{y \mid \beta_{-i}(y) \geq c-x_{2}\right\}}\left[x_{2}-c+\beta_{-i}(y)\right] f(y) d y \\
& =\left[x_{2}-\beta_{i}\left(x_{1}\right)\right] P_{1}+\int_{Y_{M} \cap\left\{y \mid \beta_{-i}(y) \geq c-x_{2}\right\}}\left[x_{2}-c+\beta_{-i}(y)\right] f(y) d y
\end{aligned}
$$

where $P_{1}=\int_{Y_{H} \cap\left\{y \mid y \geq c-\beta_{i}\left(x_{1}\right)\right\}} f(y) d y$ and $P_{2}=\int_{\left(Y_{H} \cup Y_{M}\right) \cap\left\{y \mid y \geq c-\beta_{i}\left(x_{2}\right)\right\}} f(y) d y$.

Now, $\pi^{i}\left(x_{2}, \beta_{i}\left(x_{2}\right), \beta_{-i}\right) \geq \pi^{i}\left(x_{2}, \beta_{i}\left(x_{1}\right), \beta_{-i}\right)$ implies

$$
\left[x_{2}-\beta_{i}\left(x_{2}\right)\right] P_{2} \geq\left[x_{2}-\beta_{i}\left(x_{1}\right)\right] P_{1}+\int_{Y_{M} \cap\left\{y \mid \beta_{-i}(y) \geq c-x_{2}\right\}}\left[x_{2}-c+\beta_{-i}(y)\right] f(y) d y
$$

which implies $\left[x_{2}-\beta_{i}\left(x_{2}\right)\right] P_{2} \geq\left[x_{2}-\beta_{i}\left(x_{1}\right)\right] P_{1}$, or $P_{2} \geq \frac{\left[x_{2}-\beta_{i}\left(x_{1}\right)\right]}{\left[x_{2}-\beta_{i}\left(x_{2}\right)\right]} P_{1} \cdot{ }^{19}$ Since $x_{1}<x_{2}$, we have $P_{2}>\frac{\left[x_{1}-\beta_{i}\left(x_{1}\right)\right]}{\left[x_{1}-\beta_{i}\left(x_{2}\right)\right]} P_{1}$. This follows from the fact that

$$
\frac{d}{d x}\left(\frac{\left[x-\beta_{i}\left(x_{1}\right)\right]}{\left[x-\beta_{i}\left(x_{2}\right)\right]}\right)=\frac{\left[x-\beta_{i}\left(x_{2}\right)-x+\beta_{i}\left(x_{1}\right)\right]}{\left[x-\beta_{i}\left(x_{2}\right)\right]}=\frac{\left[\beta_{i}\left(x_{1}\right)-\beta_{i}\left(x_{2}\right)\right]}{\left[x-\beta_{i}\left(x_{2}\right)\right]^{2}}>0
$$

And $P_{2}>\frac{\left[x_{1}-\beta_{i}\left(x_{1}\right)\right]}{\left[x_{1}-\beta_{i}\left(x_{2}\right)\right]} P_{1}$ implies $\pi^{i}\left(x_{1}, \beta_{i}\left(x_{2}\right), \beta_{-i}\right)>\pi^{i}\left(x_{1}, \beta_{i}\left(x_{1}\right), \beta_{-i}\right)$. Note that, we implicitly as-

\footnotetext{
${ }^{19}$ Note that $x_{2}>\beta_{i}\left(x_{2}\right)$, simply because $x_{2}>x_{1} \geq \beta_{i}\left(x_{1}\right)>\beta_{i}\left(x_{2}\right)$.
} 
sumed that $P_{1}>0$. However, if $P_{1}=0$, then $\pi^{i}\left(x_{1}, \beta_{i}\left(x_{1}\right), \beta_{-i}\right)=0$ which contradicts with Lemma 2.

(2) $c-x_{1}>\beta_{i}\left(x_{1}\right)>\beta_{i}\left(x_{2}\right)>c-x_{2}$. The relevant payoffs are

$$
\begin{gathered}
\pi^{i}\left(x_{1}, \beta_{i}\left(x_{1}\right), \beta_{-i}\right)=\left[x_{1}-\beta_{i}\left(x_{1}\right)\right] P_{1} \\
\pi^{i}\left(x_{2}, \beta_{i}\left(x_{2}\right), \beta_{-i}\right)=\left[x_{2}-\beta_{i}\left(x_{2}\right)\right] P_{2}+\int_{Y_{L} \cap\left\{y \mid \beta_{-i}(y) \geq c-x_{2}\right\}}\left[x_{2}-c+\beta_{-i}(y)\right] f(y) d y \\
\pi^{i}\left(x_{1}, \beta_{i}\left(x_{2}\right), \beta_{-i}\right)=\left[x_{1}-\beta_{i}\left(x_{2}\right)\right] P_{2}+\int_{Y_{L} \cap\left\{y \mid \beta_{-i}(y) \geq c-x_{1}\right\}}\left[x_{1}-c+\beta_{-i}(y)\right] f(y) d y \\
\pi^{i}\left(x_{2}, \beta_{i}\left(x_{1}\right), \beta_{-i}\right)=\left[x_{2}-\beta_{i}\left(x_{1}\right)\right] P_{1}+\int_{Y_{M} \cup\left(Y_{L} \cap\left\{y \mid \beta_{-i}(y) \geq c-x_{2}\right\}\right)}\left[x_{2}-c+\beta_{-i}(y)\right] f(y) d y \\
\pi^{i}\left(x_{2}, \beta_{i}\left(x_{2}\right), \beta_{-i}\right) \geq \pi^{i}\left(x_{2}, \beta_{i}\left(x_{1}\right), \beta_{-i}\right) \operatorname{implies} \\
{\left[x_{2}-\beta_{i}\left(x_{2}\right)\right] P_{2}+\int_{Y_{L} \cap\left\{y \mid \beta_{-i}(y) \geq c-x_{2}\right\}}\left[x_{2}-c+\beta_{-i}(y)\right] f(y) d y} \\
\geq\left[x_{2}-\beta_{i}\left(x_{1}\right)\right] P_{1}+\int_{Y_{M} \cup\left(Y_{L} \cap\left\{y \mid \beta_{-i}(y) \geq c-x_{2}\right\}\right)}\left[x_{2}-c+\beta_{-i}(y)\right] f(y) d y
\end{gathered}
$$

which implies $\left[x_{2}-\beta_{i}\left(x_{2}\right)\right] P_{2} \geq\left[x_{2}-\beta_{i}\left(x_{1}\right)\right] P_{1}+\int_{Y_{M}}\left[x_{2}-c+\beta_{-i}(y)\right] f(y) d y$. Hence $\left[x_{2}-\beta_{i}\left(x_{2}\right)\right] P_{2} \geq$ $\left[x_{2}-\beta_{i}\left(x_{1}\right)\right] P_{1}$. And by a similar argument in case 1 above, we get $\left[x_{1}-\beta_{i}\left(x_{2}\right)\right] P_{2}>\left[x_{1}-\beta_{i}\left(x_{1}\right)\right] P_{1}$, which in turn implies

$$
\left[x_{1}-\beta_{i}\left(x_{2}\right)\right] P_{2}+\int_{Y_{L} \cap\left\{y \mid \beta_{-i}(y) \geq c-x_{1}\right\}}\left[x_{1}-c+\beta_{-i}(y)\right] f(y) d y>\left[x_{1}-\beta_{i}\left(x_{1}\right)\right] P_{1}
$$

that is, $\pi^{i}\left(x_{1}, \beta_{i}\left(x_{2}\right), \beta_{-i}\right)>\pi^{i}\left(x_{1}, \beta_{i}\left(x_{1}\right), \beta_{-i}\right){ }^{20}$

(3) $\beta_{i}\left(x_{1}\right)>c-x_{1}>c-x_{2}>\beta_{i}\left(x_{2}\right)$. The relevant payoffs are

$$
\begin{aligned}
\pi^{i}\left(x_{1}, \beta_{i}\left(x_{1}\right), \beta_{-i}\right) & =\left[x_{1}-\beta_{i}\left(x_{1}\right)\right] P_{1}+\int_{Y_{M} \cap\left\{y \mid \beta_{-i}(y) \geq c-x_{1}\right\}}\left[x_{1}-c+\beta_{-i}(y)\right] f(y) d y \\
\pi^{i}\left(x_{2}, \beta_{i}\left(x_{2}\right), \beta_{-i}\right) & =\left[x_{2}-\beta_{i}\left(x_{2}\right)\right] P_{2} \\
\pi^{i}\left(x_{1}, \beta_{i}\left(x_{2}\right), \beta_{-i}\right) & =\left[x_{1}-\beta_{i}\left(x_{2}\right)\right] P_{2} \\
\pi^{i}\left(x_{2}, \beta_{i}\left(x_{1}\right), \beta_{-i}\right) & =\left[x_{2}-\beta_{i}\left(x_{1}\right)\right] P_{1}+\int_{Y_{M} \cap\left\{y \mid \beta_{-i}(y) \geq c-x_{2}\right\}}\left[x_{2}-c+\beta_{-i}(y)\right] f(y) d y
\end{aligned}
$$

\footnotetext{
${ }^{20}$ In case $P_{1}=0$, then $\pi^{i}\left(x_{1}, \beta_{i}\left(x_{1}\right), \beta_{-i}\right)=0$. Hence the argument given in case 1 applies.
} 
$\pi^{i}\left(x_{2}, \beta_{i}\left(x_{2}\right), \beta_{-i}\right) \geq \pi^{i}\left(x_{2}, \beta_{i}\left(x_{1}\right), \beta_{-i}\right)$ implies

$$
\begin{aligned}
& {\left[x_{2}-\beta_{i}\left(x_{2}\right)\right] P_{2} \geq\left[x_{2}-\beta_{i}\left(x_{1}\right)\right] P_{1}+\int_{Y_{M} \cap\left\{y \mid \beta_{-i}(y) \geq c-x_{2}\right\}}\left[x_{2}-c+\beta_{-i}(y)\right] f(y) d y } \\
\Rightarrow \quad & P_{2} \geq \frac{\left[x_{2}-\beta_{i}\left(x_{1}\right)\right]}{\left[x_{2}-\beta_{i}\left(x_{2}\right)\right]} P_{1}+\frac{1}{\left[x_{2}-\beta_{i}\left(x_{2}\right)\right]} \int_{Y_{M} \cap\left\{y \mid \beta_{-i}(y) \geq c-x_{2}\right\}}\left[x_{2}-c+\beta_{-i}(y)\right] f(y) d y \\
\Rightarrow \quad & P_{2}>\frac{\left[x_{1}-\beta_{i}\left(x_{1}\right)\right]}{\left[x_{1}-\beta_{i}\left(x_{2}\right)\right]} P_{1}+\frac{1}{\left[x_{2}-\beta_{i}\left(x_{2}\right)\right]} \int_{Y_{M} \cap\left\{y \mid \beta_{-i}(y) \geq c-x_{2}\right\}}\left[x_{2}-c+\beta_{-i}(y)\right] f(y) d y \\
\Rightarrow \quad & {\left[x_{1}-\beta_{i}\left(x_{2}\right)\right] P_{2}>\left[x_{1}-\beta_{i}\left(x_{1}\right)\right] P_{1}+\frac{\left[x_{1}-\beta_{i}\left(x_{2}\right)\right]}{\left[x_{2}-\beta_{i}\left(x_{2}\right)\right]} \int_{Y_{M} \cap\left\{y \mid \beta_{-i}(y) \geq c-x_{2}\right\}}\left[x_{2}-c+\beta_{-i}(y)\right] f(y) d y } \\
\Rightarrow \quad & {\left[x_{1}-\beta_{i}\left(x_{2}\right)\right] P_{2}>\left[x_{1}-\beta_{i}\left(x_{1}\right)\right] P_{1}+\frac{\left[x_{1}-\beta_{i}\left(x_{2}\right)\right]}{\left[x_{2}-\beta_{i}\left(x_{2}\right)\right]} \int_{Y_{M} \cap\left\{y \mid \beta_{-i}(y) \geq c-x_{2}\right\}}\left[x_{2}-c+\beta_{-i}(y)\right] f(y) d y } \\
& +\int_{Y_{M} \cap\left\{y \mid \beta_{-i}(y) \geq c-x_{1}\right\}}\left[x_{1}-c+\beta_{-i}(y)\right] f(y) d y-\int_{Y_{M} \cap\left\{y \mid \beta_{-i}(y) \geq c-x_{1}\right\}}\left[x_{1}-c+\beta_{-i}(y)\right] f(y) d y \\
\Rightarrow \quad & \pi^{i}\left(x_{1}, \beta_{i}\left(x_{2}\right)\right)>\pi^{i}\left(x_{1}, \beta_{i}\left(x_{1}\right)\right)+\frac{\left[x_{1}-\beta_{i}\left(x_{2}\right)\right]}{\left[x_{2}-\beta_{i}\left(x_{2}\right)\right]} \int_{Y_{M} \cap\left\{y \mid \beta_{-i}(y) \geq c-x_{2}\right\}}\left[x_{2}-c+\beta-i(y)\right] f(y) d y \\
& -\int_{Y_{M} \cap\left\{y \mid \beta_{-i}(y) \geq c-x_{1}\right\}}\left[x_{1}-c+\beta_{-i}(y)\right] f(y) d y
\end{aligned}
$$

We are done if we can show

$$
\int_{Y_{M} \cap\left\{y \mid \beta_{-i}(y) \geq c-x_{2}\right\}} \frac{\left[x_{2}-c+\beta_{-i}(y)\right]}{\left[x_{2}-\beta_{i}\left(x_{2}\right)\right]} f(y) d y \geq \int_{Y_{M} \cap\left\{y \mid \beta_{-i}(y) \geq c-x_{1}\right\}} \frac{\left[x_{1}-c+\beta_{-i}(y)\right]}{\left[x_{1}-\beta_{i}\left(x_{2}\right)\right]} f(y) d y
$$

First we will show that for any set of values $Y \subseteq Y_{M}$, we have

$$
\int_{Y} \frac{\left[x_{2}-c+\beta_{-i}(y)\right]}{\left[x_{2}-\beta_{i}\left(x_{2}\right)\right]} f(y) d y>\int_{Y} \frac{\left[x_{1}-c+\beta_{-i}(y)\right]}{\left[x_{1}-\beta_{i}\left(x_{2}\right)\right]} f(y) d y
$$

To see this,

$$
\begin{aligned}
\frac{d}{d x} \int_{Y} \frac{\left[x-c+\beta_{-i}(y)\right]}{\left[x-\beta_{i}\left(x_{2}\right)\right]} f(y) d y & =\int_{Y} \frac{d}{d x} \frac{\left[x-c+\beta_{-i}(y)\right]}{\left[x-\beta_{i}\left(x_{2}\right)\right]} f(y) d y \\
& =\int_{Y} \frac{\left[x-\beta_{i}\left(x_{2}\right)-x+c-\beta_{-i}(y)\right]}{\left[x-\beta_{i}\left(x_{2}\right)\right]^{2}} f(y) d y \\
& =\int_{Y} \frac{\left[c-\beta_{i}\left(x_{2}\right)-\beta_{-i}(y)\right]}{\left[x-\beta_{i}\left(x_{2}\right)\right]^{2}} f(y) d y \\
& >0
\end{aligned}
$$


To see the last inequality, note that for any $y \in Y_{M}$ we have $\beta_{-i}(y)<\beta_{i}\left(x_{1}\right) \leq x_{1}$ and $\beta_{i}\left(x_{2}\right) \leq$ $c-x_{2}<c-x_{1}$. Hence for any $y \in Y_{M}$, we have $\beta_{i}\left(x_{2}\right)+\beta_{-i}(y)<c$. To conclude,

$$
\begin{aligned}
\int_{Y_{M} \cap\left\{y \mid \beta_{-i}(y) \geq c-x_{2}\right\}} \frac{\left[x_{2}-c+\beta_{-i}(y)\right]}{\left[x_{2}-\beta_{i}\left(x_{2}\right)\right]} f(y) d y & >\int_{Y_{M} \cap\left\{y \mid \beta_{-i}(y) \geq c-x_{2}\right\}} \frac{\left[x_{1}-c+\beta_{-i}(y)\right]}{\left[x_{1}-\beta_{i}\left(x_{2}\right)\right]} f(y) d y \\
& \geq \int_{Y_{M} \cap\left\{y \mid \beta_{-i}(y) \geq c-x_{1}\right\}} \frac{\left[x_{1}-c+\beta_{-i}(y)\right]}{\left[x_{1}-\beta_{i}\left(x_{2}\right)\right]} f(y) d y
\end{aligned}
$$

The last inequality above follows from $Y_{M} \cap\left\{y \mid \beta_{-i}(y) \geq c-x_{1}\right\} \subseteq Y_{M} \cap\left\{y \mid \beta_{-i}(y) \geq c-x_{2}\right\} .{ }^{21}$

(4) $\beta_{i}\left(x_{1}\right)>c-x_{1}>\beta_{i}\left(x_{2}\right)>c-x_{2}$. The relevant payoffs are

$$
\begin{gathered}
\pi^{i}\left(x_{1}, \beta_{i}\left(x_{1}\right), \beta_{-i}\right)=\left[x_{1}-\beta_{i}\left(x_{1}\right)\right] P_{1}+\int_{Y_{M} \cap\left\{y \mid \beta_{-i}(y) \geq c-x_{1}\right\}}\left[x_{1}-c+\beta_{-i}(y)\right] f(y) d y \\
\pi^{i}\left(x_{2}, \beta_{i}\left(x_{2}\right), \beta_{-i}\right)=\left[x_{2}-\beta_{i}\left(x_{2}\right)\right] P_{2}+\int_{Y_{L} \cap\left\{y \mid \beta_{-i}(y) \geq c-x_{2}\right\}}\left[x_{2}-c+\beta_{-i}(y)\right] f(y) d y \\
\pi^{i}\left(x_{1}, \beta_{i}\left(x_{2}\right), \beta_{-i}\right)=\left[x_{1}-\beta_{i}\left(x_{2}\right)\right] P_{2} \\
\pi^{i}\left(x_{2}, \beta_{i}\left(x_{1}\right), \beta_{-i}\right)=\left[x_{2}-\beta_{i}\left(x_{1}\right)\right] P_{1}+\int_{Y_{M} \cup\left(Y_{L} \cap\left\{y \mid \beta_{-i}(y) \geq c-x_{2}\right\}\right)}\left[x_{2}-c+\beta_{-i}(y)\right] f(y) d y \\
\pi^{i}\left(x_{2}, \beta_{i}\left(x_{2}\right), \beta_{-i}\right) \geq \pi^{i}\left(x_{2}, \beta_{i}\left(x_{1}\right), \beta_{-i}\right) \operatorname{implies} \\
{\left[x_{2}-\beta_{i}\left(x_{2}\right)\right] P_{2}+\int_{Y_{L} \cap\left\{y \mid \beta_{-i}(y) \geq c-x_{2}\right\}}\left[x_{2}-c+\beta_{-i}(y)\right] f(y) d y} \\
\geq\left[x_{2}-\beta_{i}\left(x_{1}\right)\right] P_{1}+\int_{Y_{M} \cup\left(Y_{L} \cap\left\{y \mid \beta_{-i}(y) \geq c-x_{2}\right\}\right)}\left[x_{2}-c+\beta_{-i}(y)\right] f(y) d y
\end{gathered}
$$

\footnotetext{
${ }^{21}$ If $P_{1}=0$, then the proof still works because of the strict inequality

$\int_{Y_{M} \cap\left\{y \mid \beta_{-i}(y) \geq c-x_{2}\right\}} \frac{\left[x_{2}-c+\beta_{-i}(y)\right]}{\left[x_{2}-\beta_{i}\left(x_{2}\right)\right]} f(y) d y>\int_{Y_{M} \cap\left\{y \mid \beta_{-i}(y) \geq c-x_{1}\right\}} \frac{\left[x_{1}-c+\beta_{-i}(y)\right]}{\left[x_{1}-\beta_{i}\left(x_{2}\right)\right]} f(y) d y$
} 


$$
\begin{aligned}
\Rightarrow & {\left[x_{2}-\beta_{i}\left(x_{2}\right)\right] P_{2} \geq\left[x_{2}-\beta_{i}\left(x_{1}\right)\right] P_{1}+\int_{Y_{M}}\left[x_{2}-c+\beta_{-i}(y)\right] f(y) d y } \\
\Rightarrow & P_{2} \geq \frac{\left[x_{2}-\beta_{i}\left(x_{1}\right)\right]}{\left[x_{2}-\beta_{i}\left(x_{2}\right)\right]} P_{1}+\frac{1}{\left[x_{2}-\beta_{i}\left(x_{2}\right)\right]} \int_{Y_{M}}\left[x_{2}-c+\beta_{-i}(y)\right] f(y) d y \\
\Rightarrow & P_{2}>\frac{\left[x_{1}-\beta_{i}\left(x_{1}\right)\right]}{\left[x_{1}-\beta_{i}\left(x_{2}\right)\right]} P_{1}+\frac{1}{\left[x_{2}-\beta_{i}\left(x_{2}\right)\right]} \int_{Y_{M}}\left[x_{2}-c+\beta_{-i}(y)\right] f(y) d y \\
\Rightarrow \quad & {\left[x_{1}-\beta_{i}\left(x_{2}\right)\right] P_{2}>\left[x_{1}-\beta_{i}\left(x_{1}\right)\right] P_{1}+\frac{\left[x_{1}-\beta_{i}\left(x_{2}\right)\right]}{\left[x_{2}-\beta_{i}\left(x_{2}\right)\right]} \int_{Y_{M}}\left[x_{2}-c+\beta_{-i}(y)\right] f(y) d y } \\
& \left.+\int_{Y_{M} \cap\left\{y \mid \beta_{-i}(y) \geq c-x_{1}\right\}}\left[x_{1}-c+\beta_{-i}(y)\right] f(y) d y-\int_{Y_{M} \cap\left\{y \mid \beta_{-i}(y) \geq c-x_{1}\right\}}-c+\beta_{-i}(y)\right] f(y) d y \\
\Rightarrow \quad & \pi^{i}\left(x_{1}, \beta_{i}\left(x_{2}\right), \beta_{-i}\right)>\pi^{i}\left(x_{1}, \beta_{i}\left(x_{1}\right), \beta_{-i}\right)+\frac{\left[x_{1}-\beta_{i}\left(x_{2}\right)\right]}{\left[x_{2}-\beta_{i}\left(x_{2}\right)\right]} \int_{Y_{M}}\left[x_{2}-c+\beta_{-i}(y)\right] f(y) d y \\
& -\int_{Y_{M}}\left[x_{1}-c+\beta_{-i}(y)\right] f(y) d y
\end{aligned}
$$

Since $\beta_{i}\left(x_{2}\right)<c-x_{1}$, for any $y \in Y_{M}$, we have $\beta_{i}\left(x_{2}\right)+\beta_{-i}(y)<c$. So for any $Y \subseteq Y_{M}$,

$$
\int_{Y} \frac{\left[x_{2}-c+\beta_{-i}(y)\right]}{\left[x_{2}-\beta_{i}\left(x_{2}\right)\right]} f(y) d y>\int_{Y} \frac{\left[x_{1}-c+\beta_{-i}(y)\right]}{\left[x_{1}-\beta_{i}\left(x_{2}\right)\right]} f(y) d y
$$

Hence $\pi^{i}\left(x_{1}, \beta_{i}\left(x_{2}\right), \beta_{-i}\right)>\pi^{i}\left(x_{1}, \beta_{i}\left(x_{1}\right), \beta_{-i}\right) .{ }^{22}$

(5) $\beta_{i}\left(x_{1}\right)>\beta_{i}\left(x_{2}\right)>c-x_{1}>c-x_{2}$. Since $\bar{\beta}_{i} \leq c / 2$ and $\beta_{-i}(y)<\beta_{i}\left(x_{1}\right)$ for any $y \in Y_{M}$ we have $\beta_{i}\left(x_{2}\right)+\beta_{-i}(y)<c$ for any $y \in Y_{M}$. Then the argument given in case 4 above proves $\pi^{i}\left(x_{1}, \beta_{i}\left(x_{2}\right), \beta_{-i}\right)>\pi^{i}\left(x_{1}, \beta_{i}\left(x_{1}\right), \beta_{-i}\right) .{ }^{23}$

\footnotetext{
${ }^{22}$ If $P_{1}=0$, the argument given in previous footnote works here too.

${ }^{23}$ To see this,

$$
\begin{gathered}
\pi^{i}\left(x_{1}, \beta_{i}\left(x_{1}\right), \beta_{-i}\right)=\left[x_{1}-\beta_{i}\left(x_{1}\right)\right] P_{1}+\int_{Y_{M} \cup\left(Y_{L} \cap\left\{y \mid \beta_{-i}(y) \geq c-x_{1}\right\}\right)}\left[x_{1}-c+\beta_{-i}(y)\right] f(y) d y \\
\pi^{i}\left(x_{2}, \beta_{i}\left(x_{2}\right), \beta_{-i}\right)=\left[x_{2}-\beta_{i}\left(x_{2}\right)\right] P_{2}+\int_{Y_{L} \cap\left\{y \mid \beta_{-i}(y) \geq c-x_{2}\right\}}\left[x_{2}-c+\beta_{-i}(y)\right] f(y) d y \\
\pi^{i}\left(x_{1}, \beta_{i}\left(x_{2}\right), \beta_{-i}\right)=\left[x_{1}-\beta_{i}\left(x_{2}\right)\right] P_{2}+\int_{Y_{L} \cap\left\{y \mid \beta_{-i}(y) \geq c-x_{1}\right\}}\left[x_{1}-c+\beta_{-i}(y)\right] f(y) d y \\
\pi^{i}\left(x_{2}, \beta_{i}\left(x_{1}\right), \beta_{-i}\right)=\left[x_{2}-\beta_{i}\left(x_{1}\right)\right] P_{1}+\int_{Y_{M} \cup\left(Y_{L} \cap\left\{y \mid \beta_{-i}(y) \geq c-x_{2}\right\}\right)}\left[x_{2}-c+\beta_{-i}(y)\right] f(y) d y
\end{gathered}
$$
}

where $P_{1}=\int_{Y_{H} \cap\left\{y \mid y \geq c-\beta_{i}\left(x_{1}\right)\right\}} f(y) d y$ and $P_{2}=\int_{Y_{M} \cap\left\{y \mid y \geq c-\beta_{i}\left(x_{2}\right)\right\}} f(y) d y$.Again, $\pi^{i}\left(x_{2}, \beta_{i}\left(x_{2}\right), \beta_{-i}\right) \geq$ $\pi^{i}\left(x_{2}, \beta_{i}\left(x_{1}\right), \beta_{-i}\right)$ implies

$$
\begin{aligned}
& {\left[x_{2}-\beta_{i}\left(x_{2}\right)\right] P_{2}+\int_{Y_{L} \cap\left\{y \mid \beta_{-i}(y) \geq c-x_{2}\right\}}\left[x_{2}-c+\beta_{-i}(y)\right] f(y) d y } \\
\geq & {\left[x_{2}-\beta_{i}\left(x_{1}\right)\right] P_{1}+\int_{Y_{M} \cup\left(Y_{L} \cap\left\{y \mid \beta_{-i}(y) \geq c-x_{2}\right\}\right)}\left[x_{2}-c+\beta_{-i}(y)\right] f(y) d y }
\end{aligned}
$$




\section{Appendix B}

Lemma 8 Consider a non-trivial equilibrium, $\left(\beta_{A}(\cdot), \beta_{B}(\cdot)\right)$, with non-decreasing bidding functions. Then $\pi^{i}\left(x, \beta_{i}(x), \beta_{-i}\right)>0$ for all $x \in[c-1,1]$, for $i=A, B$.

Proof. Similar to the proof of Lemma 2 .

We will first assume that for $i=A, B, \beta_{i}$ is non-decreasing over $[0,1]$ where $\bar{\beta}_{i}$ is finite, and show that there is no non-trivial equilibrium within this domain. Later on, we will generalize this result by allowing $\bar{\beta}_{i}$ to be infinite. Then we will consider the bidding functions which are decreasing over a set of values, and show that there is no such non-trivial equilibrium. Finally we will rule out any non-trivial constant bidding. Hence all equilibria will be trivial.

Lemma 9 Consider a non-trivial equilibrium, $\left(\beta_{A}(\cdot), \beta_{B}(\cdot)\right)$, with non-decreasing bidding functions, where $\bar{\beta}_{i}$ is finite. Then $\bar{\beta}_{i} \leq c / 2$ for $i=A, B$.

Proof. Suppose $\bar{\beta}_{i}>c / 2$. Then we will consider two cases: $\bar{\beta}_{-i}>c / 2$ and $c / 2 \geq \bar{\beta}_{-i}$.

(1) $\bar{\beta}_{-i}>c / 2$ :

If $\bar{\beta}_{-i}>\bar{\beta}_{i}$, then bidder $i$, who drops out at $\bar{\beta}_{i}$ (or arbitrarily close to $\bar{\beta}_{i}$, in case there is no value for which he drops out at $\bar{\beta}_{i}$ ) would deviate to $\bar{\beta}_{-i}+\varepsilon$ where $\varepsilon>0$ is arbitrarily small. To see that this is a profitable deviation, note that for those bids of bidder $-i$ which are already smaller than $\bar{\beta}_{i}$, bidder $i$ 's payoff is not affected. This is because once he is the second one to drop out, his payoff is

$$
\begin{aligned}
& \Rightarrow \quad\left[x_{2}-\beta_{i}\left(x_{2}\right)\right] P_{2} \geq\left[x_{2}-\beta_{i}\left(x_{1}\right)\right] P_{1}+\int_{Y_{M}}\left[x_{2}-c+\beta_{-i}(y)\right] f(y) d y \\
& \Rightarrow \quad P_{2} \geq \frac{\left[x_{2}-\beta_{i}\left(x_{1}\right)\right]}{\left[x_{2}-\beta_{i}\left(x_{2}\right)\right]} P_{1}+\frac{1}{\left[x_{2}-\beta_{i}\left(x_{2}\right)\right]} \int_{Y_{M}}\left[x_{2}-c+\beta_{-i}(y)\right] f(y) d y \\
& \Rightarrow \quad P_{2}>\frac{\left[x_{1}-\beta_{i}\left(x_{1}\right)\right]}{\left[x_{1}-\beta_{i}\left(x_{2}\right)\right]} P_{1}+\frac{1}{\left[x_{2}-\beta_{i}\left(x_{2}\right)\right]} \int_{Y_{M}}\left[x_{2}-c+\beta_{-i}(y)\right] f(y) d y \\
& \Rightarrow \quad\left[x_{1}-\beta_{i}\left(x_{2}\right)\right] P_{2}>\left[x_{1}-\beta_{i}\left(x_{1}\right)\right] P_{1}+\frac{\left[x_{1}-\beta_{i}\left(x_{2}\right)\right]}{\left[x_{2}-\beta_{i}\left(x_{2}\right)\right]} \int_{Y_{M}}\left[x_{2}-c+\beta_{-i}(y)\right] f(y) d y \\
& \quad \mp \int_{Y_{M} \cap\left\{y \mid \beta_{-i}(y) \geq c-x_{1}\right\}}\left[x_{1}-c+\beta_{-i}(y)\right] f(y) d y \\
& \Rightarrow \quad \pi\left(x_{1}, \beta\left(x_{2}\right), \beta_{-i}\right) \geq\left[x_{1}-\beta\left(x_{2}\right)\right] P_{2}>\pi\left(x_{1}, \beta\left(x_{1}\right), \beta_{-i}\right) \\
&+\frac{\left[x_{1}-\beta\left(x_{2}\right)\right]}{\left[x_{2}-\beta\left(x_{2}\right)\right]} \int_{Y_{M}}\left[x_{2}-c+\beta_{-i}(y)\right] f(y) d y-\int_{Y_{M}}\left[x_{1}-c+\beta_{-i}(y)\right] f(y) d y
\end{aligned}
$$

Since $\bar{\beta}_{i} \leq c / 2$ and $\beta_{-i}(y)<\beta_{i}\left(x_{1}\right)$ for any $y \in Y_{M}$ we have $\beta_{i}\left(x_{2}\right)+\beta_{-i}(y)<c$ for any $y \in Y_{M}$. Hence for any $Y \subseteq Y_{M}$, we have

$$
\int_{Y} \frac{\left[x_{2}-c+\beta_{-i}(y)\right]}{\left[x_{2}-\beta_{i}\left(x_{2}\right)\right]} f(y) d y>\int_{Y} \frac{\left[x_{1}-c+\beta_{-i}(y)\right]}{\left[x_{1}-\beta_{i}\left(x_{2}\right)\right]} f(y) d y
$$

Therefore as in case 4 we have $\pi^{i}\left(x_{1}, \beta_{i}\left(x_{2}\right), \beta_{-i}\right)>\pi^{i}\left(x_{1}, \beta_{i}\left(x_{1}\right), \beta_{-i}\right)$. 
independent of his drop out price. And for those bids of bidder $-i$, which are bigger than $\bar{\beta}_{i}$, deviating to $\bar{\beta}_{-i}+\varepsilon$ makes him strictly better off since he will guarantee to be the second bidder to drop out. And since $\bar{\beta}_{-i} \geq \bar{\beta}_{i}>c / 2$, his payment is $c-\bar{\beta}_{-i}<c / 2$, which is less than what he would have paid if he were to drop out first, which is strictly bigger than $c / 2$. If $\bar{\beta}_{i}>\bar{\beta}_{-i}$, then bidder $-i$ deviates to $\bar{\beta}_{i}+\varepsilon$ by the same reasoning given above. If $\bar{\beta}_{i}=\bar{\beta}_{-i}$, then there is deviation by either one of the bidders.

(2) $c / 2 \geq \bar{\beta}_{-i}:$ First, we rule out the case where $\bar{\beta}_{-i}=c / 2$. To show that there is a profitable deviation, define $\widetilde{x}$ to be the value of bidder $i$ where $\beta_{i}(x)<c / 2$ for all $x<\widetilde{x}$, and $\beta_{i}(x)>c / 2$ for all $x>\widetilde{x}$. Note that bidder $i$ has a positive measure of values for which he bids less than $c / 2$, because otherwise bidder $-i$ bidding $\bar{\beta}_{-i}$ would simply deviate to any $b$ such that $b>\bar{\beta}_{i}$. Consider two sub-cases, $\widetilde{x}>c / 2$, and $\widetilde{x} \leq c / 2$.

When $\widetilde{x}>c / 2$, bidder $-i$ with value 1 deviates to $c / 2-\varepsilon$ which is profitable. To see this, first assume that $\beta_{i}($.$) is continuous at \widetilde{x}$. Define $\widetilde{\widetilde{x}}$ to be the value such that $\beta_{i}(x)<c / 2-\varepsilon$ for all $x<\widetilde{\widetilde{x}}$, and $c / 2>\beta_{i}(x) \geq c / 2-\varepsilon$ for all $\widetilde{x} \geq x \geq \widetilde{\widetilde{x}}$. In the range $[0, \widetilde{\widetilde{x}}]$ the payoffs from bidding $c / 2$ and $c / 2-\varepsilon$ are the same, so we just need to look at the range $[\widetilde{\widetilde{x}}, 1]$. The payoff from bidding $c / 2-\varepsilon$, over $[\widetilde{\widetilde{x}}, 1]$, is

$$
\int_{\widetilde{\widetilde{x}}}^{1}[1-c / 2+\varepsilon] f(x) d x=\int_{\widetilde{\widetilde{x}}}^{\widetilde{x}}[1-c / 2+\varepsilon] f(x) d x+\int_{\widetilde{x}}^{1}[1-c / 2+\varepsilon] f(x) d x
$$

And the payoff from bidding $c / 2$, over $[\widetilde{\widetilde{x}}, 1]$, is

$$
\int_{\widetilde{\widetilde{x}}}^{\widetilde{x}}\left[1-c+\beta_{i}(x)\right] f(x) d x-\int_{\widetilde{x}}^{1}(1-c / 2) f(x) d x
$$

Therefore the net gain from deviation is

$$
\int_{\widetilde{\widetilde{x}}}^{\widetilde{x}}\left[c / 2+\varepsilon-\beta_{i}(x)\right] f(x) d x+\int_{\widetilde{x}}^{1} \varepsilon f(x) d x>0
$$

The strict inequality follows from the fact that $\beta_{i}(x) \leq c / 2$ for $\widetilde{x}>x>\widetilde{\widetilde{x}}$. If $\beta_{i}($.$) is discontinuous at$ $\widetilde{x}$, then $\widetilde{x}=\widetilde{\widetilde{x}}$. Hence the net gain is simply $\int_{\widetilde{x}}^{1} \varepsilon f(x) d x>0$.

When $\widetilde{x} \leq c / 2$, bidder $-i$ with value 1 deviates to any $b$ such that $b>\bar{\beta}_{i}$. This deviation is profitable because of the following reason. Over the range $[0, \widetilde{x}]$, there is no gain or loss, and over the range $[\widetilde{x}, c / 2]$ the net gain is non-negative. However, over the range $[c / 2,1]$ the net gain is strictly positive by a similar argument given in case 1 above. 
Now we focus on the case where $\bar{\beta}_{-i}<c / 2$. Note that $\bar{\beta}_{-i}>c-1$ by definition. Then bidder $i$ with value $\widehat{x}$ deviates to $\bar{\beta}_{-i}-\varepsilon$, where $\widehat{x} \in\left\{x \mid \beta_{i}(x)>\bar{\beta}_{j}\right\}$. To see that this is a profitable deviation:

$$
\pi^{i}\left(\widehat{x}, \beta_{i}(\widehat{x}), \beta_{-i}\right)=\int_{\left\{y \mid \widehat{x} \geq c-\beta_{-i}(y)\right\}}\left[\widehat{x}-c+\beta_{-i}(y)\right] f(y) d y
$$

and

$$
\pi^{i}\left(\widehat{x}, \bar{\beta}_{-i}-\varepsilon, \beta_{-i}\right)=\int_{\left\{y \mid \widehat{x} \geq c-\beta_{-i}(y)\right\} \backslash Y_{\varepsilon}}\left[\widehat{x}-c+\beta_{-i}(y)\right] f(y) d y+\int_{Y_{\varepsilon} \cap\left\{y \mid y \geq c-\bar{\beta}_{-i}+\varepsilon\right\}}\left[\widehat{x}-\bar{\beta}_{-i}+\varepsilon\right] f(y) d y
$$

where $Y_{\varepsilon}=\left\{y \mid \bar{\beta}_{-i}>\beta_{-i}(y)>\bar{\beta}_{-i}-\varepsilon\right\}$. Note that definition of $\bar{\beta}_{-i}$ implies that $Y_{\varepsilon}$ has a positive measure for arbitrarily small $\varepsilon$. Hence $Y_{\varepsilon} \subseteq\left\{y \mid y \geq c-\bar{\beta}_{-i}+\varepsilon\right\}$. To show this, let $y \in Y_{\varepsilon}$. Since $\beta_{j}($. is non-decreasing and $\left\{y \mid \bar{\beta}_{-i}=\beta_{-i}(y)\right\}$ has measure-zero, ${ }^{24}$ we can pick $\varepsilon$ arbitrarily small so that $y$ is arbitrarily close to 1 . Since $\bar{\beta}_{-i}>c-1$, we also have $1>c-\bar{\beta}_{-i}+\varepsilon$. Then $y>c-\bar{\beta}_{-i}+\varepsilon$. Hence $y \in\left\{y \mid y \geq c-\bar{\beta}_{-i}+\varepsilon\right\}$. Therefore $Y_{\varepsilon} \cap\left\{y \mid y \geq c-\bar{\beta}_{-i}+\varepsilon\right\}=Y_{\varepsilon}$. Then, the net gain is

$$
\begin{aligned}
\pi^{i}\left(\widehat{x}, \bar{\beta}_{-i}-\varepsilon, \beta_{-i}\right)-\pi^{i}\left(\widehat{x}, \beta_{i}(\widehat{x}), \beta_{-i}\right) & =\int_{Y_{\varepsilon}}\left[\widehat{x}-\bar{\beta}_{-i}+\varepsilon-\widehat{x}+c-\beta_{-i}(y)\right] f(y) d y \\
& =\int_{Y_{\varepsilon}}\left[c-\bar{\beta}_{-i}-\beta_{-i}(y)+\varepsilon\right] f(y) d y>0
\end{aligned}
$$

since $c / 2>\bar{\beta}_{-i}>\beta_{-i}(y)$ for all $y \in Y_{\varepsilon}$.

Assuming that $\beta_{i}(x)$ is non-decreasing, define $x^{i}$ to be the value such that $\beta_{i}(x) \geq c-x^{i}$ for all $x>x^{i}$, and $\beta_{i}(x) \leq c-x^{i}$ for all $x<x^{i}$. And let $b^{i}=c-x^{i} \cdot y^{-i}$ and $b^{-i}$ are defined analogously.

Lemma 10 Consider a non-trivial equilibrium, $\left(\beta_{A}(\cdot), \beta_{B}(\cdot)\right)$, with non-decreasing bidding functions, where $\bar{\beta}_{i}$ is finite. Then $\beta_{i}(x) \leq b^{-i}$ for all $x \in[0,1]$, for $i=A, B$.

Proof. Similar to the proof of Lemma 4.

Lemma 11 There exists no non-trivial equilibrium, $\left(\beta_{A}(\cdot), \beta_{B}(\cdot)\right)$, with non-decreasing bidding functions where $\bar{\beta}_{i}$ is finite.

Proof. Similar to the proof of Lemma 5.

\footnotetext{
${ }^{24}$ If $\left\{y \mid \bar{\beta}_{-i}=\beta_{-i}(y)\right\}$ has a positive measure, then bidder $i$ would simply deviate to $\bar{\beta}_{-i}$ and hence match the bid of bidder $-i$ with positive probability which would be a profitable deviation, since $\bar{\beta}_{-i}<c / 2$.
} 
Having showed that there is no non-trivial equilibrium with non-decreasing bidding functions where $\bar{\beta}_{i}$ is finite for $i=A, B$, now we focus on bidding functions that are decreasing over some set of values.

Lemma 12 Consider a non-trivial equilibrium, $\left(\beta_{A}(\cdot), \beta_{B}(\cdot)\right)$. Assume $\bar{\beta}_{i} \leq c / 2$, for $i=A, B$. Then for any $x_{1}, x_{2} \in(c-1,1]$ such that $x_{1}<x_{2}$, if $\beta_{i}\left(x_{1}\right) \leq x_{1}$ and $\beta_{i}\left(x_{2}\right) \leq x_{2}$, then $\beta_{i}\left(x_{1}\right) \leq \beta_{i}\left(x_{2}\right)$, for $i=A, B$.

Proof. Similar to the proof of Lemma 6 .

Lemma 13 There exists no non-trivial equilibrium in which $\beta^{i}(x)>\bar{\beta}_{j}$ for $x \in X_{+}$where $X_{+}$is a positive measure set.

Proof. Suppose that in a non-trivial equilibrium $\beta_{i}(x)>\bar{\beta}_{-i}$ for a set of values with positive measure. Consider two cases, $\bar{\beta}_{i}$ is either finite or infinite.

case(1) $\bar{\beta}_{i}$ is finite: If $\bar{\beta}_{-i} \geq c / 2$, bidder $-i$ with a value $y$ such that $\beta_{-i}(y)=\bar{\beta}_{-i}$ deviates to a bid that is higher than $\bar{\beta}_{i}$. If there is no such $y$, then the same deviation will work for bidder $-i$ with a value for which he bids arbitrarily close to $\bar{\beta}_{-i}$. This deviation does not affect his payoff for bidder $i$ 's bids that are less than $\bar{\beta}_{-i}$. However, it generates strictly higher payoff for all $x$ such that $\beta_{i}(x)>\bar{\beta}_{-i}$ since $c-\beta_{i}(x)<\bar{\beta}_{-i}$. Hence the net gain is positive since the set of such $x$ has positive measure by assumption. Therefore, $\bar{\beta}_{-i}<c / 2$. First note that if $\beta_{-i}(\widehat{y})>\widehat{y}$ for some $\widehat{y}$ then $\left\{x \mid \beta_{i}(x)>\beta_{-i}(\widehat{y})\right\}$ must be a measure-zero set, because otherwise he would drop out at a sufficiently higher price to avoid non-positive payoffs. Since $\left\{x \mid \beta_{i}(x)>\beta_{-i}(y)\right\}$ has a positive measure for any $y$, we must have $\beta_{-i}(y) \leq y$ for all $y$. Since $\bar{\beta}_{-i}<c / 2$, Lemma 14 implies that $\beta_{-i}($.$) cannot be strictly decreasing$ over any range. But now we are back in case 2 in Lemma 11, where bidder $i$ with value $\widehat{x}$ deviates to $\bar{\beta}_{-i}-\varepsilon$, where $\widehat{x} \in\left\{x \mid \beta_{i}(x)>\bar{\beta}_{-i}\right\}$.

case $(2) \bar{\beta}_{i}=\infty$.

When $\bar{\beta}_{-i}<c / 2$ the proof in case 1 still applies since it does not rely on how big $\bar{\beta}_{i}$ is. So we only need to show that whenever $\bar{\beta}_{-i} \geq c / 2$ there is a deviation. Note that bidder $-i$ cannot deviate to a bid that is higher than $\bar{\beta}_{i}$ because the probability to pay an arbitrarily large amount is positive. So, bidder $-i$ cannot have an arbitrarily large $\bar{\beta}_{-i}$. Also note that there is no $x \in[0,1]$ such that $\bar{\beta}_{-i}>\beta_{i}(x) \geq c / 2$. Otherwise, bidder $i$ would deviate to a bid that is higher than $\bar{\beta}_{-i}$.

For any $x \leq c-\bar{\beta}_{-i}$, any bid higher than $\bar{\beta}_{-i}$ yields zero payoff. However, bidding $c / 2$ would guarantee a positive expected payoff if $\bar{\beta}_{-i}>c / 2$. If $\bar{\beta}_{-i}=c / 2$, then bidding $c / 2-\nu$ would guarantee 
a positive expected payoff for sufficiently small $\nu>0$. Hence we have $\beta_{i}(x)<\bar{\beta}_{-i}$ for all $x \leq c-\bar{\beta}_{-i}$. Moreover, $\beta_{i}(x)<x$ for $x \leq c-\bar{\beta}_{-i}$. Otherwise the expected payoff is non-positive. This is because for those values $y$ such that bidder $i$ is the second one to drop out, bidder $i$ never wants to pay the rest which follows from $c-\left(c-\bar{\beta}_{-i}\right) \geq \beta_{-i}(y)$ for any $y \in[0,1]$. And for those values $y$ such that bidder $i$ is the first one to drop out, the payoff is non-positive when $\beta_{i}(x) \geq x$.

Now, consider the set $\bar{X}=\left\{x \mid \beta_{i}(x)>\bar{\beta}_{-i}\right\}$. If inf $\bar{X}>c-\bar{\beta}_{-i}$, then bidder $-i$ with bid $\bar{\beta}_{-i}$ deviates to $\bar{\beta}_{-i}-\varepsilon$ (If $\beta_{-i}(y)<\bar{\beta}_{-i}$ for all $y \in[0,1]$, then bidder $-i$ with bid $\bar{\beta}_{-i}-\varepsilon / 2$ deviates to $\left.\bar{\beta}_{-i}-\varepsilon\right)$. To see that this is a profitable deviation, first note that for any $x \in[0,1] \backslash \bar{X}, \beta_{i}(x)<c / 2$. Since $\bar{\beta}_{-i} \geq c / 2$, for small enough $\varepsilon$, we have $\bar{\beta}_{-i}-\varepsilon>\beta_{i}(x)$ for all $x \in[0,1] \backslash \bar{X}$. Hence the payoff is only affected for $x \in \bar{X}$. However, since $\inf \bar{X}>c-\bar{\beta}_{-i}$ and $\varepsilon$ is arbitrarily small, bidder $i$ will still contribute the rest for any value $x \in \bar{X}$, and hence bidder $-i$ will pay less by bidding $\bar{\beta}_{-i}-\varepsilon$, instead of $\bar{\beta}_{-i}$ without decreasing the probability of provision.

Therefore, to finish the proof, we only need to show that inf $\bar{X}=c-\bar{\beta}_{-i}$ cannot be the case. Suppose it is the case. First note that it is not the case that $\beta_{i}\left(c-\bar{\beta}_{-i}\right)>\bar{\beta}_{-i}$ because the payoff would be zero for bidder $i$, while bidding $c / 2$ would yield a positive expected payoff. Now consider $\widetilde{x} \in\left\{x \mid c-\bar{\beta}_{-i}<x<c-\bar{\beta}_{-i}+\varepsilon, \beta_{i}(x)>\bar{\beta}_{-i}\right\}$ for $\varepsilon>0$. Then $\pi^{i}\left(\widetilde{x}, \beta_{i}(\widetilde{x}), \beta_{-i}\right)$ is zero in the limit as $\varepsilon$ goes to zero. However, $\pi^{i}\left(\widetilde{x}, b, \beta_{-i}\right)>0$, where $b \in\left(c-\bar{\beta}_{-i}, c-1\right)$. To see this, first note that if the set $\left\{y \mid y \geq \bar{\beta}_{-i}, \beta_{-i}(y) \geq c-\bar{\beta}_{-i}\right\}$ has a positive measure, then $\pi^{i}\left(\widetilde{x}, b, \beta_{-i}\right)>0$ for any $b \in\left(c-\bar{\beta}_{-i}, c-1\right)$. If the set $\left\{y \mid y \geq \bar{\beta}_{-i}, \beta_{-i}(y) \geq c-\bar{\beta}_{-i}\right\}$ is a measure-zero set, then $\pi^{i}\left(\widetilde{x}, b, \beta_{-i}\right)>0$ for any $b$ such that $\inf _{\bar{\beta}_{-i} \leq y \leq 1} \beta_{-i}(y)<b<c-1$. This follows from the fact that for bidder $-i$ it is never optimal to bid $c-1$ for values $y \geq \bar{\beta}_{-i}$. To see this, note that $\left\{y \mid \beta_{-i}(y) \geq c / 2\right\}$ has a positive measure ${ }^{25}$ and $\left\{y \mid y \geq \bar{\beta}_{-i}, \beta_{-i}(y) \geq c-\bar{\beta}_{-i}\right\}$ has measure-zero. So, by the definition of $\bar{\beta}_{-i}$, there exist an $\epsilon>0$ such that for all $y \in\left(\bar{\beta}_{-i}-\epsilon, \bar{\beta}_{-i}\right)$ the bidder $-i$ bids arbitrarily close to $\bar{\beta}_{-i}$. Therefore, for any $y>\bar{\beta}_{-i}$ the payoff from bidding arbitrarily close to $\bar{\beta}_{-i}$ gives a positive payoff. Since bidding $c-1$ gives zero payoff, we have $\beta_{-i}(y)>c-1$ for all values $y \geq \bar{\beta}_{-i}$. Hence bidder $i$ with value $\widetilde{x}$ can get a positive payoff from bidding slightly above $c-1$ but below the infimum of the support of the bids of bidder $-i$ for the values in the range $y \geq \bar{\beta}_{-i}$. The payoff from such a bid is independent of $\varepsilon$, which gives us a contradiction. Therefore it must be the case that inf $\bar{X}>c-\bar{\beta}_{-i}$.

\footnotetext{
${ }^{25}$ Since $\bar{\beta}_{-i} \geq c / 2$, the set $\left\{y \mid \beta_{-i}(y) \geq c / 2\right\}$ has a positive measure. Otherwise we would be back in the case with $\bar{\beta}_{-i}<c / 2$.
} 
Note that, above lemma has also showed that there exists no equilibrium, $\left(\beta_{A}(\cdot), \beta_{B}(\cdot)\right)$, where $\bar{\beta}_{i}$ is infinite, $i=A, B$. Hence we have,

Lemma 14 There exists no non-trivial equilibrium, $\left(\beta_{A}(\cdot), \beta_{B}(\cdot)\right)$, with non-decreasing bidding functions.

Proof. Follows from Lemma 13 and the fact that in any equilibrium $\bar{\beta}_{i}$ is finite for $i=A, B$.

Suppose that $\beta_{i}(x)>x$ for a positive measure set of values $X_{+}$. Then it must be the case that $\beta_{i}(x)>\bar{\beta}_{-i}$ for all $x \in X_{+}$, Otherwise bidder $i$ can simply deviate to a bid that is higher than $\bar{\beta}_{-i}$ and hence avoid negative payoffs. ${ }^{26}$ Also for those bids of bidder $-i$ that are less than $\beta_{i}(x)$, bidder $i$ is still the second bidder to drop out if he deviates to a bid higher than $\bar{\beta}_{-i}$, so there is no loss. Hence it is a profitable deviation. Therefore, the set of values such that $\beta_{i}(x)>x$ has measure-zero.

We will focus on the set of values that has $\beta_{i}(x) \leq x$ in the following lemma which is essentially revisiting case 5 in Lemma 14.

Lemma 15 Consider a non-trivial equilibrium, $\left(\beta_{A}(\cdot), \beta_{B}(\cdot)\right)$. Suppose that $\beta_{i}\left(x_{1}\right)>\beta_{i}\left(x_{2}\right)$ where $x_{1}<x_{2}$, and that $\beta_{i}\left(x_{k}\right) \leq x_{k}$ for $k=1,2$. Then $\beta_{i}\left(x_{1}\right)>\bar{\beta}_{-i}$ for $i=A, B$.

Proof. The only case where we can have $\beta_{i}\left(x_{1}\right)>\beta_{i}\left(x_{2}\right)$ with $x_{1}<x_{2}$ is when $\beta_{i}\left(x_{k}\right)>c-x_{k}$ for $k=1,2$. and $\beta_{i}\left(x_{2}\right)>c-x_{1}$. This is case 5 in Lemma 14. Although Lemma 14 assumes that $\beta_{i}(x) \leq c / 2$ for all $x \in[0,1]$, cases $1-4$ do not use this assumption. Hence we need to focus on case 5 without using this assumption. Again define

$$
\begin{aligned}
Y_{H} & =\left\{y \mid \beta_{-i}(y) \geq \beta_{i}\left(x_{1}\right)\right\} \\
Y_{M} & =\left\{y \mid \beta_{i}\left(x_{1}\right)>\beta_{-i}(y) \geq \beta_{i}\left(x_{2}\right)\right\} \\
Y_{L} & =\left\{y \mid \beta_{i}\left(x_{2}\right)>\beta_{-i}(y)\right\}
\end{aligned}
$$

If $\beta_{i}\left(x_{2}\right)+\beta_{-i}(y)<c$ for any $y \in Y_{M}$, then a similar argument given in case 4 in Lemma 14 proves that $\pi^{i}\left(x_{1}, \beta_{i}\left(x_{2}\right)\right)>\pi^{i}\left(x_{1}, \beta_{i}\left(x_{1}\right)\right)$. Therefore we assume that $\beta_{i}\left(x_{2}\right)+\beta_{-i}(y) \geq c$ for some $y \in Y_{M}$ which implies $\beta_{i}\left(x_{1}\right)>c / 2$. Hence $x_{1}>c / 2$ since $\beta_{i}\left(x_{1}\right) \leq x_{1}$. The relevant payoffs are given as

\footnotetext{
${ }^{26}$ This deviation is possible since Lemma 7 showed that $\bar{\beta}_{-i}$ cannot be infinite.
} 
follows.

$$
\begin{gathered}
\pi^{i}\left(x_{1}, \beta_{i}\left(x_{1}\right), \beta_{-i}\right)=\left[x_{1}-\beta_{i}\left(x_{1}\right)\right] P_{1}+\int_{Y_{M} \cup\left(Y_{L} \cap\left\{y \mid \beta_{-i}(y) \geq c-x_{1}\right\}\right)}\left[x_{1}-c+\beta_{-i}(y)\right] f(y) d y \\
\pi^{i}\left(x_{2}, \beta_{i}\left(x_{2}\right), \beta_{-i}\right)=\left[x_{2}-\beta_{i}\left(x_{2}\right)\right] P_{2}+\int_{Y_{L} \cap\left\{y \mid \beta_{-i}(y) \geq c-x_{2}\right\}}\left[x_{2}-c+\beta_{-i}(y)\right] f(y) d y \\
\pi^{i}\left(x_{1}, \beta_{i}\left(x_{2}\right), \beta_{-i}\right)=\left[x_{1}-\beta_{i}\left(x_{2}\right)\right] P_{2}+\int_{Y_{L} \cap\left\{y \mid \beta_{-i}(y) \geq c-x_{1}\right\}}\left[x_{1}-c+\beta_{-i}(y)\right] f(y) d y \\
\pi^{i}\left(x_{2}, \beta_{i}\left(x_{1}\right), \beta_{-i}\right)=\left[x_{2}-\beta_{i}\left(x_{1}\right)\right] P_{1}+\int_{Y_{M} \cup\left(Y_{L} \cap\left\{y \mid \beta_{-i}(y) \geq c-x_{2}\right\}\right)}\left[x_{2}-c+\beta_{-i}(y)\right] f(y) d y
\end{gathered}
$$

where $P_{1}=\int_{Y_{H} \cap\left\{y \mid y \geq c-\beta_{i}\left(x_{1}\right)\right\}} f(y) d y$ and $P_{2}=\int_{Y_{M} \cap\left\{y \mid y \geq c-\beta_{i}\left(x_{2}\right)\right\}} f(y) d y$.

$\pi^{i}\left(x_{2}, \beta_{i}\left(x_{2}\right), \beta_{-i}\right) \geq \pi^{i}\left(x_{2}, \beta_{i}\left(x_{1}\right), \beta_{-i}\right)$ implies

$$
\begin{gathered}
{\left[x_{2}-\beta_{i}\left(x_{2}\right)\right] P_{2}+\int_{Y_{L} \cap\left\{y \mid \beta_{-i}(y) \geq c-x_{2}\right\}}\left[x_{2}-c+\beta_{-i}(y)\right] f(y) d y} \\
\geq\left[x_{2}-\beta_{i}\left(x_{1}\right)\right] P_{1}+\int_{Y_{M} \cup\left(Y_{L} \cap\left\{y \mid \beta_{-i}(y) \geq c-x_{2}\right\}\right)}\left[x_{2}-c+\beta_{-i}(y)\right] f(y) d y
\end{gathered}
$$

That is,

$$
\left[x_{2}-\beta_{i}\left(x_{2}\right)\right] P_{2} \geq\left[x_{2}-\beta_{i}\left(x_{1}\right)\right] P_{1}+\int_{Y_{M}}\left[x_{2}-c+\beta_{-i}(y)\right] f(y) d y
$$

If $Y_{H} \cap\left\{y \mid y \geq c-\beta_{i}\left(x_{1}\right)\right\}$ has a positive measure, then we have

$$
\left[x_{1}-\beta_{i}\left(x_{2}\right)\right] P_{2}>\left[x_{1}-\beta_{i}\left(x_{1}\right)\right] P_{1}+\int_{Y_{M}}\left[x_{1}-c+\beta_{-i}(y)\right] f(y) d y
$$

Then,

$$
\left[x_{1}-\beta_{i}\left(x_{2}\right)\right] P_{2}>\left[x_{1}-\beta_{i}\left(x_{1}\right)\right] P_{1}+\int_{Y_{M} \cup Y_{L}}\left[x_{1}-c+\beta_{-i}(y)\right] f(y) d y-\int_{Y_{L}}\left[x_{1}-c+\beta_{-i}(y)\right] f(y) d y
$$

That is,

$$
\begin{gathered}
{\left[x_{1}-\beta_{i}\left(x_{2}\right)\right] P_{2}+\int_{Y_{L} \cap\left\{y \mid \beta_{-i}(y) \geq c-x_{1}\right\}}\left[x_{1}-c+\beta_{-i}(y)\right] f(y) d y} \\
> \\
{\left[x_{1}-\beta_{i}\left(x_{1}\right)\right] P_{1}+\int_{Y_{M} \cup\left(Y_{L} \cap\left\{y \mid \beta_{-i}(y) \geq c-x_{1}\right\}\right)}\left[x_{1}-c+\beta_{-i}(y)\right] f(y) d y}
\end{gathered}
$$


which implies $\pi^{i}\left(x_{1}, \beta_{i}\left(x_{2}\right), \beta_{-i}\right)>\pi^{i}\left(x_{1}, \beta_{i}\left(x_{1}\right), \beta_{-i}\right)$. Therefore it must be the case that $Y_{H} \cap\{y \mid y \geq$ $\left.c-\beta_{i}\left(x_{1}\right)\right\}$ is a measure-zero set. However, if $Y_{H}$ is a positive measure set, then for all $y \in Y_{H}$ we have $y<c-\beta_{i}\left(x_{1}\right)$. Since $\beta_{i}\left(x_{1}\right)>c / 2$, we have $y<c / 2$. But $\beta_{-i}(y) \geq \beta_{i}\left(x_{1}\right)$ since $y \in Y_{H}$. Therefore, $\beta_{-i}(y)>c / 2$ but $y<c / 2$. But, since the set of values with $\beta_{-i}(y)>y$ has measure-zero, $Y_{H}$ must be a measure-zero set, which implies that $\beta_{i}\left(x_{1}\right)>\bar{\beta}_{-i}$.

Lemma 16 There exists no non-trivial equilibrium with a bidding function which is decreasing over a positive measure set of values.

Proof. If there is a non-trivial equilibrium bidding function which is decreasing over a positive measure set of values $X_{+}$, then by Lemma 17 , we have $\beta_{i}(x)>\bar{\beta}_{-i}$ for all $x \in X_{+} \cdot{ }^{27}$ But then by Lemma 15 it cannot be a non-trivial equilibrium.

\footnotetext{
${ }^{27}$ Note that the set of values such that $\beta_{i}(x)>x$ has measure-zero.
} 


\section{REFERENCES}

1. Admati, A. R. and Perry, M., "Joint Projects without Commitment", The Review of Economic Studies, 58, (1991), 259-276.

2. Alboth, D., Lerner, A. and Shalev, J., "Profit Maximizing in Auctions of Public Goods", Journal of Public Economic Theory, 3 (4) (2001), 501-525.

3. Bag, P. K. and Roy, S., "On Sequential and Simultaneous Contributions under Incomplete Information" Departmental Working Papers 0805, Southern Methodist University, Department of Economics (2008).

4. Bag, P. K. and Roy, S., "Repeated Charitable Contributions under Incomplete Information" The Economic Journal, 118, (2008), 60-91.

5. Bagnoli, M. and Lipman, B. L., "Provision of Public Goods: Fully Implementing the Core through Private Contributions", The Review of Economic Studies, 56, (1989), 583-602.

6. Barbieri, S. and Malueg, D. A., "Optimal Provision of a Discrete Public Good: Linear Equilibria in the Private Information Subscription Game", Working paper (2008)

7. Barbieri, S. and Malueg, D. A., "Private Provision of a Discrete Public Good: ContinuousStrategy Equilibria in the Private-Information Subscription Game", Journal of Public Economic Theory, 10 (4) (2008), 529-545.

8. Bliss, C. and Nalebuff, B., "Dragon-Slaying and Ballroom Dancing: The Private Supply of a Public Good", Journal of Public Economics, 25, (1984), 1-12.

9. Cornelli, F., "Optimal Selling Procedures with Fixed Costs", Journal of Economic Theory, 71, (1996), 1-30.

10. Deb, R. and Razzolini, L., "Auction-Like Mechanisms for Pricing Excludable Public Goods", Journal of Economic Theory, 88, (1999), 340-368.

11. Kleindorfer, P. R. and Sertel, M. R., "Auctioning the Provision of an Indivisible Public Good" Journal of Economic Theory, 64, (1994), 20-34.

12. Krishna, V., "Auction Theory", San Diego: Academic Press (2002). 
13. Laussel, D. and Palfrey, T. R., "Efficient Equilibria in the Voluntary Contributions Mechanism with Private Information", Journal of Public Economic Theory, 5 (3) (2003), 449-478.

14. Ledyard, J. O. and Palfrey, T. R., "A Characterization of Interim Efficiency with Public Goods", Econometrica, 67 (2) (1999), 435-448.

15. Ledyard, J. O. and Palfrey, T. R., "The Approximation of Efficient Public Good by Simple Voting Schemes", Journal of Public Economics, 83 (2002), 153-171.

16. Ledyard, J. O. and Palfrey, T. R., "A General Characterization of Interim Efficient Mechanisms for Independent Linear Environments", Journal of Economic Theory, 133 (2007), 441-466.

17. Lu, J. and Quah E., "Private Provisions of a Discrete Public Good with Voluntary Participation", Journal of Public Economic Theory, 11 (3) (2009), 343-362.

18. Mailath G. J. and Postlewaite A., "Asymmetric Information Bargaining Problems with Many Agents", The Review of Economic Studies, 57, (1990), 351-367.

19. Marx, L. E. and Matthews, S. A., "Dynamic Voluntary Contribution to a Public Project", The Review of Economic Studies, 67, (2000), 327-358.

20. Menezes, F. M., Monteiro, P. K. and Temimi, A., "Private Provision of Discrete Public Goods with Incomplete Information", Journal of Mathematical Economics, 35, (2001), 493-514.

21. Morelli, M. and Vesterlund, L., "Provision Point Mechanisms and Over Provision of Public Goods", Ohio State University Working Paper \#00-14, (2000).

22. Moulin, H., "Serial Cost Sharing of Excludable Public Goods", The Review of Economic Studies, 61, (1994), 305-325.

23. Myerson, R. B., "Optimal Auction Design", Mathematics of Operations Research, 6, (1981), $58-73$

24. Varian, H. R., "Sequential Contributions to Public Goods", Journal of Public Economics, 53, (1994), 165-186. 